\title{
THE REPRESENTATION POLYHEDRON OF A SEMIORDER*
}

\author{
BARRY BALOF ${ }^{\dagger}$, JEAN-PAUL DOIGNON $^{\ddagger}$, AND SAMUEL FIORINI ${ }^{\S}$
}

\begin{abstract}
Let a finite semiorder, or unit interval order, be given. All its numerical representations (when suitably defined) form a convex polyhedron. We show that the facets of the representation polyhedron correspond to the noses and hollows of the semiorder. Our main result is to prove that the coordinates of the vertices and the components of the extreme rays of the polyhedron are all integral multiples of a common value. The result follows from the total dual integrality of the system defining the representation polyhedron. Total dual integrality is in turn derived from a particular property of the oriented cycles in the directed graph of noses and hollows of a strictly upper diagonal step tableau. Our approach delivers also a new proof for the existence of the minimal representation, a concept originally discovered by Pirlot (1990). Finding combinatorial interpretations of the vertices and extreme rays of the representation polyhedron is left for future work.
\end{abstract}

Key words. Semiorder, representation polyhedron, total dual integrality

AMS subject classifications. 06A07, 90C10, 52B12

1. Introduction. Throughout the paper, let $X$ be a finite set of $n$ elements. Suppose we assign to each element $i$ an interval $\left[x_{i}, x_{i}+r\right]$ of the real axis, with $r$ a nonnegative real value called the threshold. This naturally determines a binary relation $P$ on $X$, with $i P j$ if and only if the interval for $i$ is entirely to the left of the interval for $j$ and disjoint from it, that is,

$$
i P j \Longleftrightarrow x_{i}+r<x_{j} \quad \text { for all } i, j \in X \text {. }
$$

A binary relation on $X$ is called a semiorder whenever it can be generated from a collection of constant length intervals as described above. Because one may always set $r=1$, semiorders are also called unit interval orders. We give an example in Figure 1.1.

Semiorders have a long history (see for instance the report by Fishburn and Monjardet [12] on three papers published by Wiener in the 1910's). They play an important role in the theory of utility because they model strict preference on alternatives while allowing for intransitive indifference (cf. [13, 22]). Representing semiorders by constant-length intervals amounts to assign utility values to the alternatives and to set a threshold (the interval length) which is used when comparing two such values. For various reasons exposed in Pirlot [20], it is important to better understand the structure of the set of all representations. Taking a polyhedral point of view, we contribute towards a satisfactory answer to this question.

Consider a semiorder $P$ on $X$ generated by a collection $\left(\left[x_{i}, x_{i}+r\right]\right)_{i \in X}$ of intervals. From now on, we assume that all intervals are contained in the nonnegative real halfline. This mild condition guarantees that the polyhedra we are studying are

*This work was partially supported by a Crédit d'ouverture internationale from ULB and an Actions de Recherche Concertées (ARC) fund of the Communauté française de Belgique. We thank our colleagues Thomas McCormick, Gautier Stauffer and András Sebö for discussions on the paper and related literature. Version date: August 18, 2008

${ }^{\dagger}$ Department of Mathematics, Whitman College, Walla Walla, WA 99362, U.S.A. (balofba@whitman.edu).

${ }^{\ddagger}$ Université Libre de Bruxelles, Département de Mathématique, c.p. 216, 1050 Bruxelles, Belgium (doignon@ulb.ac.be).

$\S$ Université Libre de Bruxelles, Département de Mathématique, c.p. 216, 1050 Bruxelles, Belgium (sfiorini@ulb.ac.be). 


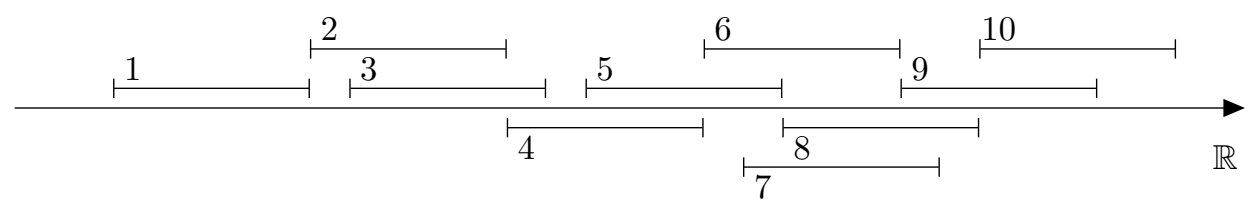

\begin{tabular}{|c|c|c|c|c|c|c|c|c|c|c|}
\hline & 1 & 2 & 3 & 4 & 5 & 6 & 7 & 8 & 0 & 10 \\
\hline 1 & 0 & 0 & 1 & 1 & 1 & 1 & 1 & 1 & 1 & 1 \\
\hline 2 & 0 & 0 & 0 & 0 & 1 & 1 & 1 & 1 & 1 & 1 \\
\hline 3 & 0 & 0 & 0 & 0 & \begin{tabular}{|l}
1 \\
\end{tabular} & 1 & 1 & 1 & 1 & 1 \\
\hline 4 & 0 & 0 & 0 & 0 & 0 & 0 & 1 & 1 & 1 & 1 \\
\hline 5 & 0 & 0 & 0 & 0 & 0 & 0 & 0 & 0 & 1 & 1 \\
\hline 6 & 0 & 0 & 0 & 0 & 0 & 0 & 0 & 0 & 0 & 1 \\
\hline 7 & 0 & 0 & 0 & 0 & 0 & 0 & 0 & 0 & 0 & \begin{tabular}{|l|}
1 \\
\end{tabular} \\
\hline 8 & 0 & 0 & 0 & 0 & 0 & 0 & 0 & 0 & 0 & 0 \\
\hline 9 & 0 & 0 & 0 & 0 & 0 & 0 & 0 & 0 & 0 & 0 \\
\hline 10 & 0 & 0 & 0 & 0 & 0 & 0 & 0 & 0 & 0 & 0 \\
\hline
\end{tabular}

Fig. 1.1. A collection of ten intervals of constant length on the real line, and a tableau for the resulting semiorder (the meaning of boxed entries is introduced in Section 2).

pointed (i.e., contain no lines; for polyhedra, we generally follow the terminology of Schrijver [23] or Ziegler [28]).

It is natural to encode the collection $\left(\left[x_{i}, x_{i}+r\right]\right)_{i \in X}$ as a pair $(x, r) \in \mathbb{R}_{+}^{X} \times \mathbb{R}_{+}$ where $x \in \mathbb{R}_{+}^{X}$ is a vector recording the position of the left endpoint of each interval and $r \in \mathbb{R}_{+}$is the threshold. Any pair $(x, r) \in \mathbb{R}_{+}^{X} \times \mathbb{R}_{+}$satisfying (1.1) is referred to as a (numerical) representation of the semiorder $P$. We denote by $\mathcal{R}$ the set of all representations of $P$. Thus the set $\mathcal{R}$ is a convex subset of the nonnegative orthant of $\mathbb{R}^{X} \times \mathbb{R}$, but in general it is neither closed nor open. In this work, we do not study $\mathcal{R}$ directly but a family of mutually homothetic polyhedra that approximate it.

Because $X$ is a finite set, for each representation $(x, r)$ of $P$ there exists some positive real number $\varepsilon$ such that

$$
\left\{\begin{array}{rll}
i P j & \Longrightarrow & x_{i}+r+\varepsilon \leq x_{j}, \\
\neg i P j & \Longrightarrow & x_{i}+r \geq x_{j}, \\
& x_{i} \geq 0,
\end{array} \quad \text { for all } i, j \in X .\right.
$$

For a given positive $\varepsilon$, we denote by $\mathcal{R}_{\varepsilon}$ the set of all pairs $(x, r) \in \mathbb{R}_{+}^{X} \times \mathbb{R}_{+}$satisfying (1.2). Those pairs are the $\varepsilon$-representations of the semiorder $P$. Letting $\varepsilon$ take any positive value, we get an easy result.

Proposition 1.1. Let $P$ be a semiorder on $X$. For any positive real number $\varepsilon$, the set $\mathcal{R}_{\varepsilon}$ of all $\varepsilon$-representations of $P$ is a pointed polyhedron in $\mathbb{R}^{X} \times \mathbb{R}$. Moreover, we have

$$
\begin{aligned}
\frac{\varepsilon^{\prime}}{\varepsilon} \mathcal{R}_{\varepsilon} & =\mathcal{R}_{\varepsilon^{\prime}} \quad \text { when } \quad \varepsilon, \varepsilon^{\prime}>0 ; \\
\mathcal{R}_{\varepsilon} & \subseteq \mathcal{R}_{\varepsilon^{\prime}} \quad \text { when } \quad \varepsilon \geq \varepsilon^{\prime}>0 ; \\
\mathcal{R} & =\cup \uparrow\left\{\mathcal{R}_{\varepsilon}: \varepsilon>0\right\} .
\end{aligned}
$$


All polyhedra $\mathcal{R}_{\varepsilon}$ are thus homothetic to one another. We call them generically the representation polyhedron of the semiorder $P$. Our main goal is to describe the geometric structure of the representation polyhedron. The so-called 'minimal $\varepsilon^{-}$ representation', whose existence is established by Pirlot [20], gives a particular point in the polyhedron $\mathcal{R}_{\varepsilon}$. However, little further is known about the representation polyhedron.

We first indicate how the facets of the polyhedron $\mathcal{R}_{\varepsilon}$ are related to the 'noses' and 'hollows' of the semiorder. Then we show that all coordinates of vertices of $\mathcal{R}_{\varepsilon}$ are integral multiples of $\varepsilon$. We also get a stronger property for the extreme rays of $\mathcal{R}_{\varepsilon}$.

Our analysis of the geometric structure of $\mathcal{R}_{\varepsilon}$ heavily relies on linear programming and network flow techniques motivated by the 'fringe graph' of the semiorder, a directed graph built from the noses and hollows (for graphs, we generally adhere to the terminology of Bang-Jensen and Gutin [2]). In particular, we establish a special property of the fringe graph which is in fact a property of $0-1$ matrices having a specific pattern. In order to provide a coherent and self-contained approach to the fringe graph, we give in passing alternative proofs for several known results on the combinatorial structure of a semiorder.

Our approach leads to a new proof for the existence of the minimal $\varepsilon$-representation, which is a special vertex of $\mathcal{R}_{\varepsilon}$. Finding combinatorial interpretations of the other vertices and also of the extreme rays of $\mathcal{R}_{\varepsilon}$ is left as an open problem for further work.

2. Background on semiorders. To define semiorders, we adopted in Section 1 the representation viewpoint based on intervals (cf. Luce [17]). Semiorders can also be characterized in a purely combinatorial way. The result, due to Scott and Suppes [25], is captured in the second condition in Proposition 2.1 below. Still another characterization is in terms of specific 'tableaux' and appears as the third condition below; it is due to Sharp [26] and Mirkin [18].

We refer the reader to Monjardet [19] for an overview of early work on semiorders, and to Fishburn [13] and Pirlot and Vincke [22] for a general exposition (including most of the results recalled in this section).

Let again $P$ be a relation on $X$. Whenever a linear ordering $L$ of $X$ is chosen, we can describe $P$ in a binary matrix $M$ whose rows as well as columns are indexed by elements of $X$ listed in accordance with $L$, by setting

$$
M_{i, j}:= \begin{cases}1 & \text { if } i P j \\ 0 & \text { otherwise. }\end{cases}
$$

Such a tableau is indexed by $L$. It is a strictly upper triangular step tableau, or for short a step tableau, when it has nondecreasing rows, nonincreasing columns and zeroes on the diagonal. Figure 1.1 shows a collection of constant length intervals together with a step tableau for the resulting semiorder. The tableau is indexed by the linear ordering $1<2<\ldots<10$.

Proposition 2.1. The following three conditions on a relation $P$ on $X$ are equivalent:

(i) $P$ is a semiorder, that is, $P$ admits a representation $(x, r) \in \mathbb{R}_{+}^{X} \times \mathbb{R}_{+}$as in (1.1) (or, equivalently, an $\varepsilon$-representation as in (1.2) for some or any positive $\varepsilon)$; 
(ii) $P$ is irreflexive and satisfies for all $i, j, k, \ell \in X$ :

$$
\begin{aligned}
& i P j \text { and } k P \ell \Longrightarrow i P \ell \text { or } k P j, \\
& i P j \text { and } j P k \Longrightarrow i P \ell \text { or } \ell P k ;
\end{aligned}
$$

(iii) $P$ admits a step tableau (for some linear ordering of $X$ ).

Linear orderings as in Proposition 2.1(iii) are said to be compatible with the semiorder $P$. The trace of $P$ is the weak order (complete preorder) $T$ on $X$ with $i T j$ whenever $x_{i} \leq x_{j}$ for at least one $\varepsilon$-representation $(x, r)$. The trace $T$ can be equivalently defined as follows, where $i, j \in X$ :

$$
i T j \Longleftrightarrow \begin{cases}j P k \Rightarrow i P k, & \forall k \in X ; \\ \ell P i \Rightarrow \ell P j, & \forall \ell \in X .\end{cases}
$$

Moreover, the trace is the union of the compatible linear orderings, and any linear ordering of $X$ contained in the trace is compatible. There exists an $\varepsilon$-representation $(x, r)$ of $P$ as in (1.2) that additionally satisfies $i T j$ if and only if $x_{i} \leq x_{j}$.

The trace $T$ of $P$, being a weak order, is paramount to an ordered partition of $X$ into classes, that we call the classes of $P$ : Two elements $i$ and $j$ fall in a same class when $i T j$ and $j T i$ and are then equivalent, denoted as $i \sim j$. The class of an element $i$ is written as $C_{i}$. We call $C_{i}$ the initial (resp. final) class if $i T j$ (resp. $j T i$ ) holds for all elements $j$ in $X$; the elements in the initial class are initial (resp. final). Notice that a semiorder $P$ has only one class if and only if $P=\varnothing$, and that $P$ has exactly two classes if and only if $P=C \times D$ for some ordered bipartition $(C, D)$ of $X$.

The semiorder $P$ is reduced when its trace $T$ is a linear ordering. When $P$ is not reduced, it is natural to define a quotient relation $P / \sim$ on the quotient set $X / \sim$. Then $P / \sim$ is a reduced semiorder on $X / \sim$ called the reduced quotient of $P$.

We need two additional notions about semiorders. First defined by Pirlot [20] (in terms similar to Proposition 3.2(iii) below), they were given an alternative, general definition by Doignon and Falmagne [10]. A pair $(a, b)$ is a nose of the semiorder $P$ on $X$ if $(a, b) \in P$ and $P \backslash\{(a, b)\}$ is again a semiorder; similarly, $(c, d)$ is a hollow of $P$ if $(c, d) \in(X \times X) \backslash P$ and $P \cup\{(c, d)\}$ is a semiorder ${ }^{1}$. Notice that, using Proposition 2.1(ii), the definitions of noses and hollows can be restated directly in terms of $P$ (see [10]). The semiorder in Figure 1.1 is reduced; in the tableau shown, its noses are circled and its hollows are boxed.

3. Noses and hollows versus facets. We start the investigation of the representation polyhedron $\mathcal{R}_{\varepsilon}$. Our first result states that $\mathcal{R}_{\varepsilon}$ has the same dimension as the space in which it is defined. In other words, $\mathcal{R}_{\varepsilon}$ has dimension $n+1$, where $n=|X|$.

Proposition 3.1. The representation polyhedron of any semiorder on $X$ is fulldimensional.

Proof. Take any nonnegative $\varepsilon-$ representation $(x, r)$ of the semiorder $P$ on $X$. By translating all intervals $\left[x_{i}, x_{i}+r\right]$ to positive values, we may assume that $x_{i}>0$ holds for all $i \in X$. Now pick some nonnegative $r^{\prime}$ and positive $\varepsilon^{\prime}$ such that $r^{\prime}+\varepsilon^{\prime}<r+\varepsilon$ and $r^{\prime}>r$. Then $\left(x, r^{\prime}\right)$ is an $\varepsilon^{\prime}$-representation satisfying all inequalities of (1.2) strictly, and also $r^{\prime}>0$. It follows that $\mathcal{R}_{\varepsilon^{\prime}}$ is full-dimensional. Hence, by Proposition 1.1, $\mathcal{R}_{\varepsilon}$ is full-dimensional too.

\footnotetext{
${ }^{1}$ In Pirlot $[20,21]$, it is $(d, c)$ that is called a hollow.
} 
Next we look at facets of $\mathcal{R}_{\varepsilon}$. Recall that an inequality in a given linear description of a full-dimensional polyhedron is irredundant if and only if it is facet-defining.

Proposition 3.2. Let $P$ be a semiorder on $X$. Then the following are equivalent for a pair $(a, b) \in X \times X$ :

(i) $a P b$ and the inequality $x_{a}+r+\varepsilon \leq x_{b}$ defines a facet of $\mathcal{R}_{\varepsilon}$;

(ii) $(a, b)$ is a nose;

(iii) in some step tableau for $P$, the cell indexed by $(a, b)$ has no 1 neither to its left nor below it.

Similarly, the following are equivalent for a pair $(c, d) \in X \times X$ with $c \neq d$ :

(iv) $\neg c P d$ and the inequality $x_{c}+r \geq x_{d}$ defines a facet of $\mathcal{R}_{\varepsilon}$;

(v) $(c, d)$ is a hollow;

(vi) in some step tableau for $P$, the cell indexed by $(c, d)$ has no 0 neither to its right nor above it.

Proof. (iii) $\Rightarrow$ (ii). In a tableau as in (iii), replace the 1 in the cell indexed by $(a, b)$ with a 0 . The resulting tableau is still strictly upper diagonal stepwise. In other words, $P \backslash\{(a, b)\}$ is a semiorder (Proposition 2.1(iii)). So $(a, b)$ is a nose.

(ii) $\Rightarrow$ (i). Any $\varepsilon$-representation of the semiorder $P \backslash\{(a, b)\}$ provides a solution to the system obtained by deleting inequality $x_{a}+r+\varepsilon \leq x_{b}$ from (1.2). The representation does not satisfy the latter inequality because it satisfies $x_{a}+r \geq x_{b}$. Thus, the former inequality is irredundant in (1.2) and hence defines a facet of the full-dimensional polyhedron $\mathcal{R}_{\varepsilon}$.

(i) $\Rightarrow$ (iii). Assume inequality $x_{a}+r+\varepsilon \leq x_{b}$ defines a facet of $\mathcal{R}_{\varepsilon}$. Among all linear orderings of $X$ compatible with $P$, select one, say $L$, that ranks $a$ last in its class and $b$ first in its class. We check that (iii) is satisfied for the tableau $M$ indexed by $L$.

To the contrary, suppose there exist elements $i, j$ such that $a L i, j L b,(i, j) \neq$ $(a, b)$ and $M_{i, j}=1$. Assume $i \neq a$ (the case $j \neq b$ is similar). Thus we have $\neg i T a$ and $x_{a}<x_{i}$ is valid for $\mathcal{R}_{\varepsilon}$. Similarly, $x_{j} \leq x_{b}$ is valid. Moreover $M_{i, j}=1$ means $i P j$ and so $x_{i}+r+\varepsilon \leq x_{j}$ is also valid. By summing the three last inequalities we infer that the original inequality $x_{a}+r+\varepsilon \leq x_{b}$ holds strictly for all points of $\mathcal{R}_{\varepsilon}$ and hence cannot be facet-defining, a contradiction.

The proof of the second part of the proposition is similar. $\square$

From Proposition 3.2 we can derive an irredundant linear description of $\mathcal{R}_{\varepsilon}$. Further interesting properties of this description are obtained below in Section 6 .

Proposition 3.3. Let $N$ and $H$ respectively denote the set of noses and hollows of the semiorder $P$ on $X$, and let $H^{-1}=\{(d, c):(c, d) \in H\}$ denote the set of hollow inverses of $P$. The polyhedron $\mathcal{R}_{\varepsilon}$ is described by the following constraints:

$$
\begin{aligned}
-x_{a}+x_{b}-r \geq \varepsilon, & \forall(a, b) \in N, \\
-x_{d}+x_{c}+r \geq 0, & \forall(d, c) \in H^{-1}, \\
x_{i} \geq 0, & \forall i \in X,
\end{aligned}
$$

Moreover, any inequality from the first two sets of constraints defines a facet. Inequality $x_{i} \geq 0$ defines a facet if and only if $i$ is an initial element. Inequality $r \geq 0$ defines a facet if and only if $P$ is a linear ordering.

Proof. The first two assertions follow at once from the previous proposition. (Notice that the inequality $r \geq 0$ in (3.1) appears several times in (1.2), under the form $x_{i}+r \geq x_{i}$.)

We now prove the "only if" part of the last two assertions. First, if $i$ is not initial then $x_{i}>0$ is valid and thus $x_{i} \geq 0$ is not facet-defining. Second, when $P$ is not 
an (irreflexive) linear ordering, there are incomparable elements $i$ and $j$ for $P$. Then adding the corresponding inequalities $x_{i}+r \geq x_{j}$ and $x_{j}+r \geq x_{i}$ in (1.2) gives $2 r \geq 0$. Thus the inequality $r \geq 0$ is redundant and hence not facet-defining.

To prove the "if" part of the last two assertions, we establish the existence of some pair $(x, r)$ in $\mathbb{R}_{+}^{X} \times \mathbb{R}_{+}$that satisfies all inequalities in (1.2) except the one under consideration. If $m$ is an initial element, there is a representation $(x, r)$ of $P$ in which $x_{m}<x_{j}$ for all elements $j \in X \backslash\{m\}$. For this representation, let $s:=x_{m}$ and $t:=\min \left\{x_{j}: j \in X \backslash\{m\}\right\}$ and define $x_{i}^{*}:=x_{i}-(s+t) / 2$. Clearly, $\left(x^{*}, r\right)$ satisfies (1.2) except for $x_{m} \geq 0$. In the case where $P$ is linear, there is a representation $(x, r)$ with $r=0$. Then for some positive real number $\eta$ taken sufficiently small, $(x,-\eta)$ satisfies (1.2) except for $r \geq 0$. $\square$

Results about the vertices and extreme rays of $\mathcal{R}_{\varepsilon}$ are less easy to obtain. To this aim, we first explore the combinatorial properties of noses and hollows in the next two sections. Consequences for the structure of $\mathcal{R}_{\varepsilon}$ will be drawn in Section 6 .

4. The fringe graph. The fringe graph of the semiorder $P$ on $X$ is the directed graph $D(P):=\left(X, N \cup H^{-1}\right)$, where $N$ and $H^{-1}$ are defined as in Proposition 3.3 above. In other words, the fringe graph has one vertex per element of the ground set, and each of its arcs is either a nose or the inverse of a hollow. Notice that $D(P)$ contains no loop and no multiple arcs, but might contain antiparallel arcs $(i, j)$ and $(j, i)$ (this happens exactly in case $j H i$ and $i H j$, and then $i, j$ must be equivalent). An example of a fringe graph is given in Figure 4.1. In Pirlot [21], such a directed graph with specific values assigned to its arcs is called the 'super synthetic graph'.

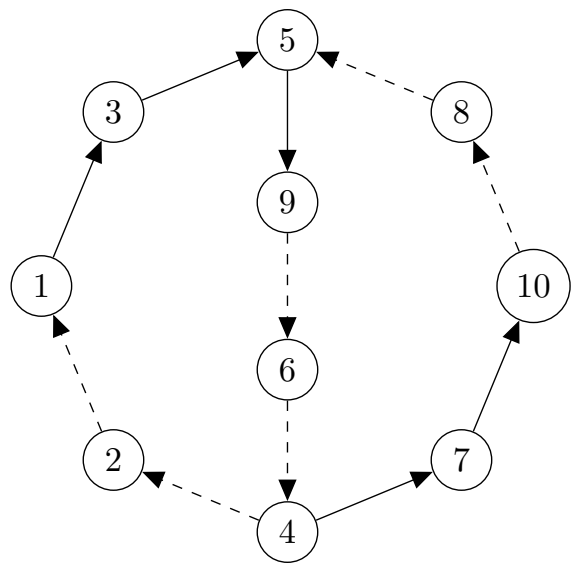

FIG. 4.1. The fringe graph for the semiorder given in Figure 1.1 (noses are indicated by plain arcs and hollow inverses by dashed arcs).

Because several of the subsequent proofs proceed by induction on the size $n$ of $X$, we examine how noses and hollows behave when elements are deleted or added. In general, the noses and hollows are not affected by the removal of elements. The addition of elements is more complicated: Even in the restricted situation we handle in Lemma 4.2, there are a few exceptional cases.

Lemma 4.1. Let $P$ be a semiorder on $X$. Consider a set $Y$ of elements to be deleted from $X$. Let $P^{\prime}$ denote the semiorder obtained by restricting $P$ to $X^{\prime}:=X \backslash Y$. Then for all $(a, b),(c, d) \in X^{\prime} \times X^{\prime}$, the following assertions are true:

(i) if $(a, b)$ is a nose of $P$, then it is a nose of $P^{\prime}$; 
(ii) if $(c, d)$ is a hollow of $P$, then it is a hollow of $P^{\prime}$.

Proof. The statements follow at once from Proposition 3.2(iii) and (vi).

Lemma 4.2. Let $P$ be a semiorder on $X$ having at least two classes, with $Y$ the final class. Let $P^{\prime}$ denote the restriction of $P$ to $X^{\prime}=X \backslash Y$ and let $\sim^{\prime}$ denote the equivalence relation of the semiorder $P^{\prime}$. For $i, j$ in $X^{\prime}$ we let $j \prec i$ if $j \sim^{\prime} i$ and $j P y$, $\neg i P y$ for all $y$ in $Y$. Then for all $(a, b),(c, d) \in X^{\prime} \times X^{\prime}$, the following assertions are true:

(i) if $(a, b)$ is a nose of $P^{\prime}$, then it is a nose of $P$ except if $e \prec b$ for some element $e$ in $X^{\prime}$. In this case (see Figure 4.2), $(a, e)$ is a nose of $P$ and $(e, y)$ a nose, $(b, y)$ a hollow of $P$ for all $y$ in $Y$;

(ii) if $(c, d)$ is a hollow of $P^{\prime}$, then it is a hollow of $P$ except in the following two cases.

CASE (II.1): there exists an element $f$ in $X^{\prime}$ such that $d \prec f$. In this case, $(c, f)$ is a hollow of $P$ and $(d, y)$ a nose, $(f, y)$ a hollow of $P$ for all $y$ in $Y$.

CASE (II.2): there exists an element $g$ in $X^{\prime}$ such that $g \prec c$. In this case, $(g, d)$ is a hollow of $P$ and $(g, y)$ a nose, $(c, y)$ a hollow of $P$ for all $y$ in $Y$.

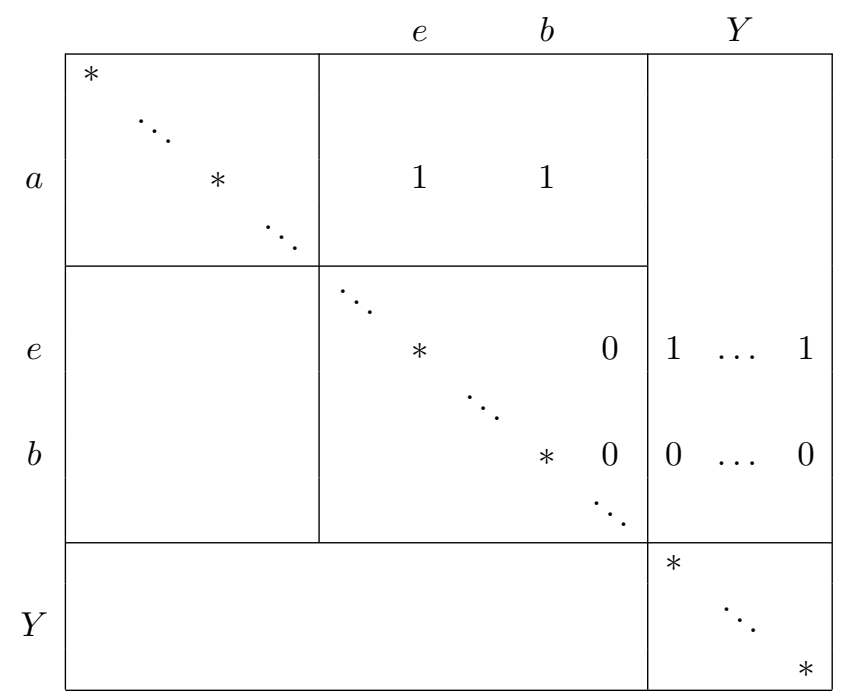

FIG. 4.2. A step tableau for a semiorder illustrating Lemma 4.2(i); the diagonal cells are marked with $*$.

Proof. As usual, let $T$ denote the trace of $P$. Similarly, let $T^{\prime}$ denote the trace of $P^{\prime}$. Consider any two elements $i, j$ in $X^{\prime}$. From (2.3) we infer that $i T j$ implies $i T^{\prime} j$ and, conversely, $i T^{\prime} j$ implies $i T j$ except if $j \prec i$. Therefore the restriction of $T$ to $X^{\prime}$ equals either $T^{\prime}$, or $T^{\prime}$ with one of its classes divided into two (this is the case in Figure 4.2, with $e \prec b$ ). Consequently, any linear ordering $\leq^{\prime}$ of $X^{\prime}$ compatible with $P^{\prime}$ extends to a linear ordering $\leq$ of $X$ compatible with $P$ having all elements of $Y$ at the end, except that maybe some elements from the divided class of $P^{\prime}$ have to be permuted among themselves. By convention, for a given $\leq^{\prime}$, we select $\leq$ in such a way as to change the initial ordering as little as possible. More precisely, we ask that the restrictions of $\leq$ and $\leq^{\prime}$ to the two classes of $P$ originating from the divided class 
of $P^{\prime}$ coincide.

It is useful to visualize how the transition from $\leq^{\prime}$ to $\leq$ affects the step tableaus. Let $M$ denote the tableau for $P$ indexed by $\leq$, and let $M^{\prime}$ denote the tableau for $P^{\prime}$ indexed by $\leq^{\prime}$. Then $M$ is obtained from $M^{\prime}$ by (A) permuting some rows and columns of $M^{\prime}$ corresponding to elements in the divided class of $P^{\prime}$ (if there is such a class) and (B) appending to the resulting tableau the rows and columns corresponding to the elements of $Y$. Notice that in the whole process the value in every cell of $M^{\prime}$ is unchanged, although the row and column labels may change. If we focus on any two particular elements $i, j$ of $X^{\prime}$ there are two possibilities for the cell indexed by $(i, j)$ in $M^{\prime}$ : Either it moves when $M^{\prime}$ is transformed into $M$ or it does not.

We now use Proposition 3.2(iii) and (vi).

First consider a nose $(a, b)$ of $P^{\prime}$. Choose the compatible linear ordering $\leq^{\prime}$ in such a way that the cell of $M^{\prime}$ indexed by $(a, b)$ has no 1 neither to its left nor below it. If the cell of $M^{\prime}$ indexed by $(a, b)$ does not move when $M^{\prime}$ is transformed into $M$ then $(a, b)$ is a nose of $P$. Otherwise, because $a P b$ and $b \in X^{\prime}$, the cell can only move to the right. In this case, $b$ belongs to the divided class of $P^{\prime}$ and there exists some element $e$ in $X^{\prime}$ such that $e \prec b$. The rest of assertion (i) follows.

Next consider a hollow $(c, d)$ of $P^{\prime}$. Choose $\leq^{\prime}$ in such a way that the cell of $M^{\prime}$ indexed by $(c, d)$ has no 0 neither to its right or above it. If the cell indexed by $(c, d)$ does not move then $(c, d)$ is a hollow of $P$ (this time we use the fact that, by hypothesis, no element of $X^{\prime}$ is equivalent to an element of $Y$ in $P$ ). Otherwise, the cell may move to the left or down. If the cell moves to the left then $d$ belongs to the divided class of $P^{\prime}$ and there exists some element $f$ in $X^{\prime}$ such that $d \prec f$. Hence Case (ii.1) occurs. Finally, if the cell moves down then $c$ belongs to the divided class of $P^{\prime}$ and there exists some element $g$ in $X^{\prime}$ such that $g \prec c$. Here Case (ii.2) occurs.

$\square$

Let $P$ be a semiorder on $X$ with trace $T$. Any pair $(a, b)$ in $P$ such that the class $C_{b}$ comes just after the class $C_{a}$ is for sure a nose; such a nose is said to be short. In this situation, we have for $i, j \in P$ that $i T a$ and $b T j$ imply $i P j$. It follows that every arc of $D(P)$ with one vertex in $U=\{i \in X: i T a\}$ and the other in $X \backslash U$ has its tail in $C_{a}$, has its head in $C_{b}$ and is a nose. Because $C_{a} \subseteq U$ and $C_{b} \subseteq X \backslash U$, we conclude that $D(P)$ is not strongly connected whenever $P$ has a short nose.

Our next result characterizes the strongly connected components of $D(P)$ as certain unions of equivalence classes of $P$. Below, we call a class $C_{b}$ leading and a class $C_{a}$ trailing whenever $(a, b)$ is a short nose of $P$. Moreover, the initial (resp. final) class of $P$ is also called leading (resp. trailing).

A directed path (resp. a directed cycle) is an oriented path (resp. an oriented cycle) in which all arcs go from one vertex to the subsequent vertex along the path (resp. cycle).

Lemma 4.3. Let $P$ be a semiorder on $X$. The strongly connected components of the fringe graph of $P$ are precisely the sets obtained as the union of all equivalence classes comprised between a leading class and the next trailing class (including these two classes). In particular, the fringe graph of $P$ is strongly connected if and only if $P$ has no short nose.

Proof. It suffices to prove the second assertion. The proof is by induction on $n$, the number of elements in the ground set $X$. The lemma trivially holds when $P$ has less than three classes. Assume now that $P$ has at least three classes and let $Y$ be the final class. As in Lemma 4.2, we set $X^{\prime}=X \backslash Y$ and denote by $P^{\prime}$ the restriction of $P$ to $X^{\prime}$. 
Take any element $y$ final for $P$. Let $k$ be any element maximal w.r.t. $T$ for satisfying $k P y$. Then $(k, y)$ is a nose of $P$. As by assumption the nose $(k, y)$ is not short, there is some class of $P$ inbetween $C_{k}$ and $Y$. Let $\ell$ belong to the class following $k$. Then $(y, \ell)$ is a hollow inverse of $P$.

By our induction assumption, $X^{\prime}$ is strongly connected in $D\left(P^{\prime}\right)$. If all noses of $P^{\prime}$ are also noses of $P$ and all hollows of $P^{\prime}$ are also hollows of $P$, then $X^{\prime}$ is also strongly connected in $D(P)$. Therefore the strong connectivity of $X$ in $D(P)$ follows in view of the nose $(k, y)$ and hollow inverse $(y, \ell)$ obtained above for every $y$ from $Y$.

In case some nose $(a, b)$ of $P^{\prime}$ is not a nose of $P$, we are in the special situation described in Lemma 4.2(i). Similarly, if some hollow $(c, d)$ of $P^{\prime}$ is not a hollow of $P$, we are in the special situation described in Lemma 4.2(ii). Using notation from the lemma, we may then in any directed path in $D\left(P^{\prime}\right)$ replace any nose $(a, b)$ of $P^{\prime}$ that is not a nose of $P$ with the three consecutive $\operatorname{arcs}(a, e)$ (nose of $P$ ), $(e, y)$ (nose of $P$ ) and $(y, b)$ (inverse hollow of $P$ ). Similarly, we may replace any hollow inverse $(d, c)$ of $P^{\prime}$ that is not a hollow inverse of $P$ with the three consecutive arcs $(d, y)$ (nose of $P),(y, f)$ (hollow inverse of $P$ ) and $(f, c)$ (hollow inverse of $P$ ) in Case (ii).1, or $(d, g)$ (hollow inverse of $P$ ),$(g, y)$ (nose of $P$ ) and $(y, c)$ (hollow inverse of $P$ ). The strong connectivity of $X$ in $D(P)$ follows again.

Lemma 4.4. Let $P$ be a semiorder on $X$. The fringe graph of $P$ is weakly connected.

Proof. The result follows from Lemma 4.3 and the existence of short noses between any strongly connected component of $D(P)$ and the next one along the trace (if the next one exists).

The following result appears in Pirlot [21]; we provide another proof of sufficiency that is more combinatorial.

Lemma 4.5. Let $P$ be a semiorder on $X$ with trace $T$, and $i, j$ two elements of $X$. Then the following three assertions are equivalent:

(i) there is a directed $i-j$ walk in the fringe graph of $P$ with at least as many noses as hollow inverses;

(ii) there is a directed $i-j$ path in the fringe graph of $P$ with at least as many noses as hollow inverses;

(iii) $i T j$ and $\neg j T i$.

Proof. (i) $\Rightarrow$ (ii). This implication follows immediately from the fact that in the fringe graph of a semiorder every directed cycle contains less noses than hollow inverses. Indeed, if we add the inequalities in (3.1) corresponding to the $\operatorname{arcs}$ of a directed cycle with at least as many noses as hollow inverses we conclude that $P$ has no representation, a contradiction.

(ii) $\Rightarrow$ (iii). If such a directed path exists, take any $\varepsilon$-representation $(x, r)$ and add all inequalities from (3.1) corresponding to noses and hollow inverses in the path. There results $-x_{i}+x_{j} \geq \alpha r+\beta \varepsilon$, where $\beta$ is the number of noses in the path and $\alpha$ the number of noses minus the number of hollow inverses. As $\alpha \geq 0$ and $\beta>0$, we get $x_{i}+\varepsilon \leq x_{j}$, and thus $i T j, \neg j T i$.

(iii) $\Rightarrow$ (i). It suffices to prove the lemma whenever $i$ and $j$ belong respectively to two successive classes. Proceeding by induction on $n$, we first note that the result trivially holds if $P$ has less than three classes. When $P$ has at least three classes, $i$ and $j$ are either both not initial or both not final. Replacing if necessary $P$ with $P^{-1}=\{(\ell, k):(k, \ell) \in P\}$, we may assume that the final class $Y$ contains neither $i$ nor $j$.

Working again with the semiorder $P^{\prime}$ induced on $X^{\prime}=X \backslash Y$, we use the induction 
hypothesis to establish the desired result. Whenever one of the special situations of Lemma 4.2 occurs, we perform the same replacement of the nose $(a, b)$ or hollow inverse $(d, c)$ of $P^{\prime}$ as we did in the proof of Lemma 4.3.

The following corollary shows that $P$ is entirely determined by $N$ and $H$. This nice property was noted by Pirlot [21] (see also Doignon and Falmagne [10]). Here is another proof of the property.

Corollary 4.6. Let $P$ be a semiorder on $X$. Then the following three assertions are equivalent:

(i) there is a directed $i-j$ walk in the fringe graph of $P$ with more noses than hollow inverses;

(ii) there is a directed $i-j$ path in the fringe graph of $P$ with more noses than hollow inverses;

(iii) $i P j$.

Proof. The proof of (i) $\Rightarrow$ (ii) is identical to the one given above in the proof of Lemma 4.5.

(ii) $\Rightarrow$ (iii). If such a directed path exists, proceed as in the previous proof. As this time $\alpha>0$ and $\beta>0$, we get $x_{i}+r+\varepsilon \leq x_{j}$, which gives $i P j$ in view of (1.2).

(iii) $\Rightarrow$ (i). As before, let $T$ denote the trace of $P$. There is necessarily a nose $(a, b)$ of $P$ such that $i T a$ and $b T j$. By Lemma 4.5, there exists an $i-a$ directed walk and a $b-j$ directed walk in $D(P)$ that both have at least as many noses as hollow inverses. Then combining these two walks with the nose $(a, b)$ we find an $i-j$ directed walk with more noses than hollow inverses in $D(P)$.

5. The oriented cycle balance lemma. Pirlot [20] shows that directed cycles in the fringe graph of a reduced semiorder have exactly one less nose than hollow inverse. Surprisingly, this very useful property can be generalized to oriented cyclesas we prove in this section.

Consider an oriented cycle $C$ in the fringe graph $D(P)$ of the semiorder $P$ on $X$. Thus $C$ is a finite alternating sequence $u_{0}, a_{1}, u_{1}, a_{2}, \ldots, u_{k-1}, a_{k}, u_{k}$ of vertices in $X$ and $\operatorname{arcs}$ in $N \cup H^{-1}$ such that, for $i=1,2, \ldots, k$, the arc $a_{i}$ equals either $\left(u_{i-1}, u_{i}\right)$ or $\left(u_{i}, u_{i-1}\right)$, and moreover the vertices $u_{0}, u_{1}, \ldots, u_{k-1}$ are distinct while $u_{k}=u_{0}$. The length of an oriented cycle is the number of arcs it contains. We define the balance of the oriented cycle $C$ as

$\left|\left\{i \in\{1,2, \ldots, k\}:\left(u_{i-1}, u_{i}\right) \in N \cup H\right\}\right|-\left|\left\{i \in\{1,2, \ldots, k\}:\left(u_{i}, u_{i-1}\right) \in N \cup H\right\}\right|$.

Assume for now that $P$ is reduced and consider a numerical representation $(x, r)$ of $P$. Then we can trace the oriented cycle $C$ in the representation by moving at each step $i=1,2, \ldots, k$ from $x_{u_{i-1}}$ to $x_{u_{i}}$ on the real line. The balance of $C$ is exactly the number of moves from left to right minus the number of moves from right to left.

The oriented cycle balance lemma (Lemma 5.2) states that the balance of an oriented cycle is always $-1,0$ or +1 , provided the arcs used satisfy a certain restriction: They should be arcs of the 'fringe graph' of some tableau of $P$. The latter restriction is always satisfied when the semiorder is reduced. (As is easily seen, when the semiorder is not reduced and the restriction not satisfied, the result may fail to be true.)

Let $M$ denote any step tableau whose rows and columns are indexed by the elements of the set $X$ listed according to some linear ordering $\leq$. Thus $\leq$ is compatible with the semiorder on $X$ represented by $M$. We say that a pair $(a, b)$ is a nose of $M$ if $M_{a, b}=1$ and $M_{i, j}=0$ for all pairs $(i, j)$ such that $a \leq i, j \leq b$ and $(i, j) \neq(a, b)$. The pair $(c, d)$ is a hollow of $M$ if $c \neq d, M_{c, d}=0$ and $M_{i, j}=1$ for all pairs $(i, j)$ such that $i \leq c, d \leq j$ and $(i, j) \neq(c, d)$. We define the fringe graph of $M$ just as we defined the 
fringe graph of a semiorder. If the semiorder $P$ on $X$ represented by the tableau $M$ is reduced, then the fringe graphs of $P$ and $M$ coincide. If $P$ is not reduced, then a nose (resp. hollow inverse) $(i, j)$ of $P$ is a nose of $M$ if and only if $i$ and $j$ are respectively the last and the first elements with respect to $\leq$ of their corresponding equivalence classes for $P$. Note the following strengthening of Lemma 4.1 in the context of step matrices.

Lemma 5.1. Let $M$ be a step tableau on $X$ indexed by the linear ordering $\leq$. Consider a set $Y$ of elements to be deleted from $X$. Let $M^{\prime}$ denote the restriction of $M$ to $X^{\prime}:=X \backslash Y$. Then for all $(a, b),(c, d) \in X^{\prime} \times X^{\prime}$, the following assertions are true:

(i) if $(a, b)$ is a nose of $M$, then $(a, b)$ is a nose of $M^{\prime}$;

(ii) if $(c, d)$ is a hollow of $M$, then $(c, d)$ is a hollow of $M^{\prime}$;

(iii) if $M_{a, b}=1$ and $Y$ contains all elements $i$ such that both $a<i$ and $M_{i, b}=1$ and $Y$ contains all elements $j$ such that both $j<b$ and $M_{a, j}=1$, then $(a, b)$ is a nose of $M^{\prime}$;

(iv) if $M_{c, d}=0, c \neq d$ and $Y$ contains all elements $i$ such that both $i<c$ and $M_{i, d}=0$ and $Y$ contains all elements $j$ such that both $d<j$ and $M_{c, j}=0$, then $(c, d)$ is a hollow of $M^{\prime}$.

We are now ready to state and prove the oriented cycle balance lemma.

Lemma 5.2 (oriented cycle balance lemma). The balance of any oriented cycle in the fringe graph of a step tableau is in $\{-1,0,1\}$.

Proof. Let $M$ be a tableau as in the statement, indexed by the linear ordering $\leq$ of $X$. Consider an oriented cycle $C$ in the fringe graph of $M$, with sequence $u_{0}, a_{1}, u_{1}$, $a_{2}, \ldots, u_{\ell-1}, a_{\ell}, u_{\ell}=u_{0}$. The proof is by induction on $n=|X|$. The key idea is to transform the oriented cycle considered to a smaller oriented cycle in the fringe graph of some smaller step tableau, without changing the balance of the oriented cycle.

The result being true for $n \leq 4$, we assume $n \geq 5$. Furthermore, we may assume that $C$ covers all elements of the ground set (that is, $\ell=n$ ), because otherwise we can delete any element not covered by $C$ and then use Lemma 5.1: as $C$ is also an oriented cycle in the fringe graph of the restricted step tableau (with the same balance as originally), we know that the balance of $C$ is in $\{-1,0,1\}$.

Now consider an element $u$ and the two $\operatorname{arcs}$ of $C$ incident to it. We say that $u$ is a right bumper if one of these arcs has head $u$ and is a nose and the other has tail $u$ and is the inverse of a hollow. In other words, $u$ is a right bumper if and only if its two neighbors on $C$ are smaller than $u$ in $\leq$. These two neighbors are necessarily consecutive in $\leq$.

Because $C$ covers all elements, the last two columns of the tableau are distinct (otherwise the last element would be covered by only one hollow and no nose), and similarly the last column contains a 0 above the diagonal and also a 1 . So the element of $X$ that is maximum for $\leq$ is a right bumper.

Without loss of generality, we may assume the following: $u_{2}$ is the right bumper that is minimum for $\leq$, we have $a_{2}=\left(u_{1}, u_{2}\right) \in N$ and $a_{3}=\left(u_{2}, u_{3}\right) \in H^{-1}$ (otherwise, we replace the oriented cycle with its reverse, this multiplies the balance of $C$ by -1 ). As noted before, $u_{1}$ and $u_{3}$ are consecutive in $\leq$.

If $a_{1}=\left(u_{0}, u_{1}\right) \in H^{-1}$ then $u_{0}$ and $u_{2}$ are consecutive in $\leq$ and the subtableau of $M$ corresponding to the four elements $u_{0}, u_{1}, u_{2}$, and $u_{3}$ looks like this (noses and 
hollows are boxed):

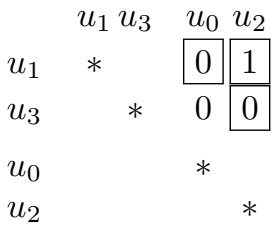

Then, by Lemma 4.1, the pair $\left(u_{3}, u_{0}\right)$ is a hollow of the tableau $M^{\prime}$ resulting from the deletion of $u_{1}$ and $u_{2}$. This means that $u_{0},\left(u_{0}, u_{3}\right), u_{3}, a_{3}, u_{4}, \ldots, u_{\ell-1}, a_{\ell}, u_{\ell}=u_{0}$ defines an oriented cycle $C^{\prime}$ in the fringe graph of $M^{\prime}$. The induction hypothesis now implies that the balance of $C^{\prime}$ is in $\{-1,0,1\}$. Because the balance of $C$ equals that of $C^{\prime}$, we are done.

Similarly, if $a_{4}=\left(u_{3}, u_{4}\right) \in N$ then $u_{2}$ and $u_{4}$ are consecutive in $\leq$. The subtableau of $M$ corresponding to the four elements $u_{1}, u_{2}, u_{3}$ and $u_{4}$ is:

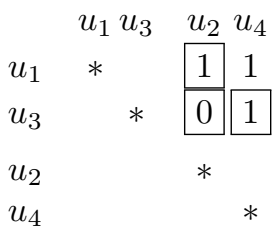

In this case we delete $u_{2}$ and $u_{3}$. This time, the pair $\left(u_{1}, u_{4}\right)$ becomes a nose. Once again we can conclude by induction.

Now suppose that we neither have $a_{1}=\left(u_{0}, u_{1}\right) \in H^{-1}$ nor $a_{4}=\left(u_{3}, u_{4}\right) \in N$. Because no two noses or hollow inverses can have the same head or tail, we have $\left(u_{0}, u_{1}\right) \in N \cup H$ and $\left(u_{4}, u_{3}\right) \in N \cup H$. It follows $u_{0}<u_{4}<u_{1}<u_{3}<u_{2}$. Remember that $u_{1}$ and $u_{3}$ are consecutive. Suppose there exists an element $u$ with $u_{0}<u<u_{4}$. Then $M_{u, u_{1}}=0$ and $M_{u, u_{3}}=1$, and thus any nose or hollow in $u$ 's row is incident to $u_{1}$ or $u_{3}$. Because $u$ is distinct from the neighbors of $u_{1}$ and $u_{3}$ on $C$ (namely, $u_{0}, u_{2}$ and $u_{4}$ ), the two $\operatorname{arcs}$ of $C$ incident to $u$ must come from $u$ 's column. It follows that $u$ is a right bumper, which contradicts the minimality of $u_{2}$. Hence $u_{0}$ and $u_{4}$ are consecutive as well.

The subtableau of $M$ corresponding to the elements $u_{i}$ with $0 \leq i \leq 4$ has the following structure:

\begin{tabular}{ccccccc} 
& $u_{0}$ & $u_{4}$ & \multicolumn{1}{c}{$u_{1}$} & $u_{3}$ & $u_{2}$ \\
$u_{0}$ & $*$ & 0 & $?$ & 1 & 1 \\
$u_{4}$ & & $*$ & & 0 & $?$ & \\
& & & & 1 \\
$u_{1}$ & & & $*$ & 0 & 1 \\
$u_{3}$ & & & & $*$ & 0 \\
$u_{2}$ & & & & & & \\
\hline
\end{tabular}

So there are four cases according to whether $\left(u_{0}, u_{1}\right)$ is a nose or a hollow and whether $\left(u_{4}, u_{3}\right)$ is a nose or a hollow. 
CASE 1. $a_{1}=\left(u_{0}, u_{1}\right) \in N$ and $a_{4}=\left(u_{4}, u_{3}\right) \in N$. In this case the subtableau is:

\begin{tabular}{|c|c|c|c|c|}
\hline & $u_{0} u_{4}$ & & & $u_{2}$ \\
\hline$u_{0}$ & $* 0$ & 1 & 1 & 1 \\
\hline$\iota_{4}$ & $*$ & 0 & 1 & 1 \\
\hline$u_{1}$ & & & 0 & 1 \\
\hline$u_{3}$ & & & $*$ & 0 \\
\hline
\end{tabular}

We can see right away that $\left(u_{1}, u_{4}\right) \in H^{-1}$. So we are done if we delete $u_{2}$ and $u_{3}$.

CASE 2. $a_{1}=\left(u_{0}, u_{1}\right) \in N$ and $a_{4}=\left(u_{3}, u_{4}\right) \in H^{-1}$. The subtableau is then:

$\begin{array}{ccccccc} & u_{0} & u_{4} & u_{1} & u_{3} & u_{2} \\ u_{0} & * & 0 & 1 & 1 & 1 \\ u_{4} & & * & 0 & 0 & 1 \\ u_{1} & & & & * & 0 & 1 \\ u_{3} & & & & * & 0 \\ u_{2} & & & & & & \\ \end{array}$

If we delete $u_{1}$ and $u_{2}$, the pair $\left(u_{0}, u_{3}\right)$ becomes a nose and we are done by induction.

CASE 3. $a_{1}=\left(u_{1}, u_{0}\right) \in H^{-1}$ and $a_{4}=\left(u_{4}, u_{3}\right) \in N$. The corresponding subtableau is:

$\begin{array}{ccccccc} & u_{0} & u_{4} & u_{1} & u_{3} & u_{2} \\ u_{0} & * & 0 & 0 & 1 & 1 \\ u_{4} & & * & 0 & 1 & 1 \\ u_{1} & & & & * & 0 & 1 \\ u_{3} & & & & * & 0 \\ u_{2} & & & & & & \\ & & & & & & \end{array}$

We delete $u_{3}$ and $u_{2}$ and then in the resulting step tableau $M^{\prime}$ change $M_{u_{0}, u_{1}}^{\prime}$ to 1 . Then $\left(u_{0}, u_{1}\right)$ becomes a nose (which replaces the hollow inverse $\left.\left(u_{1}, u_{0}\right)\right)$ and $\left(u_{1}, u_{4}\right)$ becomes a hollow inverse.

CASE 4. $a_{1}=\left(u_{1}, u_{0}\right) \in H^{-1}$ and $a_{4}=\left(u_{3}, u_{4}\right) \in H^{-1}$. In this case we have the following subtableau:

$\begin{array}{ccccccc} & u_{0} & u_{4} & u_{1} & u_{3} & u_{2} \\ u_{0} & * & 0 & 0 & 1 & 1 \\ u_{4} & & * & 0 & 0 & 1 \\ u_{1} & & & & * & 0 & 1 \\ u_{3} & & & & * & 0 \\ u_{2} & & & & & & \\ \end{array}$

This case is similar to the first case. We see right away that $\left(u_{0}, u_{3}\right)$ is a nose. So we delete $u_{1}$ and $u_{2}$ before applying the induction. 
Remark 5.1. Rephrased in terms of Gerards [14], Lemma 5.2 states that the fringe graph of a step tableau is an oriented graph of 'discrepancy' 1.

COROLLARY 5.3. The balance of any oriented cycle in the fringe graph of a reduced semiorder is in $\{-1,0,1\}$.

The oriented cycle balance lemma contains as a particular case the following result from Pirlot [20].

COROllary 5.4. Any directed cycle in the fringe graph of a reduced semiorder contains precisely one more hollow inverse than noses.

Proof. Let $P$ denote a reduced semiorder and $C$ denote a directed cycle in the fringe graph of $P$. As noted before in the proof of Lemma 4.5, the cycle $C$ contains more hollow inverses than noses. By the oriented cycle balance lemma, it follows that $C$ has exactly one more hollow inverse than nose.

The proof of the oriented cycle balance lemma can be readily adapted to prove the following result, and thus we give only a sketch of the proof.

Lemma 5.5. Let $M$ be a step tableau indexed by a linear ordering $\leq$ with initial element $m$. Any directed path in the fringe graph of $M$ starting at $m$ contains at least as many noses as hollow inverses. In particular, any directed path in the fringe graph of a reduced semiorder starting at the initial element contains at least as many noses as hollow inverses.

Proof sketch. Consider a directed path $R$ in the fringe graph of $M$ with sequence $u_{1}, a_{2}, u_{2}, a_{3}, \ldots, u_{\ell-1}, a_{\ell}, u_{\ell}$. Because $R$ is a directed path, all the $u_{i}$ 's are distinct and $a_{i}=\left(u_{i-1}, u_{i}\right)$ for $i=2, \ldots, \ell$. We proceed as in the proof of Lemma 5.2 by induction on $n$. We may assume $\ell=n \geq 5$. First notice that $a_{2}=\left(u_{1}, u_{2}\right)$ must be a nose. Second, if $R$ consists only of noses, we are done. Assume now $R$ contains also hollow inverses. Then $R$ admits at least one right bumper (we define right bumpers $u_{i}$ as in the proof of Lemma 5.2, but notice that only the case $a_{i}=\left(u_{i-1}, u_{i}\right) \in N$ and $a_{i+1}=\left(u_{i}, u_{i+1}\right) \in H^{-1}$ can occur here because the path $R$ is directed). Now consider the right bumper $u_{i}$ which is minimum for $\leq$. We must have $3 \leq i$. Moreover, if $i=\ell-1$, then we may delete $u_{i-1}, u_{i}$ and $u_{i+1}$ (and at the same time the nose $a_{i}$ and the hollow $a_{i+1}$ ), and proceed by recurrence. Hence, from now on, we assume $3 \leq i \leq \ell-2$.

The rest of the argumentation is as in the proof of Lemma 5.2, except that some cases considered there are ruled out here by the fact that $R$ is directed (namely, Cases 1, 3 and 4).

6. Consequences of the oriented cycle balance lemma. In this section, we always assume that $P$ is a semiorder on $X$, with trace $T$, nose set $N$ and hollow set $H$. We resume our investigation of the representation polyhedron $\mathcal{R}_{\varepsilon}$ of $P$. Recall from Proposition 3.3 that $\mathcal{R}_{\varepsilon}$ is the set of solutions of the system (3.1). The system is now used to write the primal in a pair of mutually dual linear programs. We assume here $w \in \mathbb{Z}^{X}, \omega \in \mathbb{Z}, y \in \mathbb{R}^{N \cup H^{-1}}$ and as usual set $y(E)=\sum_{e \in E} y_{e}$. Also, referring to the fringe graph $D(P)$, we set $\delta^{+}(i)=\left\{(k, \ell) \in N \cup H^{-1}: k=i\right\}$ and $\delta^{-}(i)=\left\{(k, \ell) \in N \cup H^{-1}: \ell=i\right\}$. The following two linear programs are mutually dual:

$$
\begin{aligned}
(\mathcal{P}) \quad \min \sum_{i \in X} w_{i} x_{i}+\omega r \quad \text { subject to }-x_{a}+x_{b}-r & \geq \varepsilon, \quad \forall(a, b) \in N, \\
-x_{d}+x_{c}+r & \geq 0, \quad \forall(d, c) \in H^{-1} \\
x_{i} & \geq 0, \quad \forall i \in X, \\
r & \geq 0 ;
\end{aligned}
$$


(D) $\max \varepsilon y(N)$ subject to $y\left(\delta^{-}(i)\right)-y\left(\delta^{+}(i)\right) \leq w_{i}, \quad \forall i \in X$,

$$
\begin{aligned}
y\left(H^{-1}\right)-y(N) & \leq \omega, \\
y_{i j} & \geq 0, \quad \forall(i, j) \in N \cup H^{-1} .
\end{aligned}
$$

Let $\mathcal{F}$ denote the feasible region of $(\mathcal{D})$, that is,

$$
\left\{y \in \mathbb{R}^{N \cup H^{-1}}: y\left(\delta^{-}(i)\right)-y\left(\delta^{+}(i)\right) \leq w_{i}, \forall i \in X ; \quad y\left(H^{-1}\right)-y(N) \leq \omega ; \quad y \geq 0\right\} .
$$

Then $\mathcal{F}$ is bounded. Indeed, since $(\mathcal{P})$ is feasible, $(\mathcal{D})$ is bounded. In other words there is a number $K$ such that $y(N) \leq K$ is valid for $\mathcal{F}$. But then $y\left(H^{-1}\right) \leq K+\omega$ is also valid for $\mathcal{F}$. It follows that $\mathcal{F}$ is bounded. We call the polytope $\mathcal{F}$ the flow polytope of $P$ (although it depends also on $w$ and $\omega$ ).

We call the constraints $y\left(\delta^{-}(i)\right)-y\left(\delta^{+}(i)\right) \leq w_{i}$ for $i \in X$ the flow balance inequalities, the constraint $y\left(H^{-1}\right)-y(N) \leq \omega$ the threshold inequality, and the contraints $y_{i j} \geq 0$ for $(i, j) \in N \cup H^{-1}$ the trivial inequalities. If we replace the less than or equal to symbol by an equality symbol in the latter constraints we obtain the flow balance equalities, threshold equality and trivial equalities.

We say that an element $i$ is tight with respect to a dual solution $y$ if $y\left(\delta^{-}(i)\right)-$ $y\left(\delta^{+}(i)\right)=w_{i}$ holds, that is, the flow balance inequality for $i$ is tight at $y$.

Lemma 6.1. Let $y$ be any optimal solution of $(\mathcal{D})$. Then every non-initial element is tight with respect to $y$.

Proof. Take an optimal solution $(x, r)$ of $(\mathcal{P})$. By complementary slackness (see e.g., [16, Section 3.4]), any non-tight element $i$ must satisfy $x_{i}=0$. Because $(x, r)$ is an $\varepsilon$-representation of our semiorder $P$, we conclude that any non-tight element is initial.

The support of a dual solution $y$ is the directed graph $S=S(y)$ with vertex set $V(S):=X$ and arc set $A(S):=\left\{(i, j) \in N \cup H^{-1}: y_{i j}>0\right\}$. Let $Z$ denote the set of elements that are not tight with respect to $y$. After contracting the vertices of $Z$ into one vertex (this may produce parallel arcs or even repeated loops), we obtain from $S$ a directed pseudograph called the reduced support of $y$ and denoted by $S^{\circ}=S^{\circ}(y)$. If $Z$ contains at most one vertex, then $S^{\circ}$ is identical to $S$. Otherwise, $S^{\circ}$ differs from $S$; we denote by $\tau$ the vertex of $S^{\circ}$ arising from the contraction of $Z$ and refer to it as the special vertex of $S^{\circ}$. By construction, there is a bijective mapping $f: A(S) \rightarrow A\left(S^{\circ}\right)$.

Now assume that $v$ is a vertex of the flow polytope $\mathcal{F}$. A standard system for $v$ is an irredundant system $B y=c$ on $\mathbb{R}^{N \cup H^{-1}}$ with unique solution $v$ and formed by first taking all trivial equalities that are satisfied by $v$, then a maximum number of flow balance equalities and finally the threshold equality if necessary.

In the statement of the next lemma and below, we consider two oriented cycles equal if they have the same arc sets. Also, oriented cycles and oriented paths in directed multigraphs are defined exactly as in directed graphs (see the beginning of Section 5).

Lemma 6.2. Let $v$ be any vertex of the flow polytope $\mathcal{F}$ with support $S$, and let $B y=c$ denote a standard system for $v$. If the reduced support $S^{\circ}$ of $v$ contains no oriented cycle, then $\operatorname{det} B= \pm 1$. Otherwise $S^{\circ}$ contains exactly one oriented cycle and $\operatorname{det} B= \pm \beta$ where $\beta$ denotes the balance of the oriented cycle.

Proof. If we order the variables $y_{i j}$ in such a way that those corresponding to arcs in $S$ come last, the coefficient matrix of the given standard system takes the following form:

$$
B=\left(\begin{array}{c|c}
I & 0 \\
\hline B_{21} & B_{22}
\end{array}\right)
$$


Obviously, det $B$ and det $B_{22}$ are equal. Moreover, because the system $B y=c$ admits the single solution $v$, both determinants are different from zero.

If the threshold equality is not part of our standard system, then $\operatorname{det} B=\operatorname{det} B_{22}=$ \pm 1 because the vertex-edge incidence matrix of a directed graph is totally unimodular, see for instance Chvátal [8], or Schrijver [24, Section 13.2].

If $S^{\circ}$ contains no oriented cycle, then $S$ contains no oriented cycle and has at most one non-tight vertex per weakly connected component. It follows that all $y_{i j}$ 's can be computed from the trivial and flow balance equalities, and so the threshold equality is not part of the standard system for $v$. From the preceding paragraph, $\operatorname{det} B=\operatorname{det} B_{22}= \pm 1$.

For the rest of the proof, we assume that the standard system involves the threshold equality and that $S^{\circ}$ contains an oriented cycle $C^{\circ}$ with $\operatorname{arc}$ set $A^{\circ}$. We write $A:=f^{-1}\left(A^{\circ}\right)$ for the set of $\operatorname{arcs}$ of $S$ mapped onto $A^{\circ}$. Notice that each arc of $A$ indexes a column of $B_{22}$. Moreover, $A$ can be regarded as either an oriented cycle, or an oriented path starting and ending at non-tight elements. We denote this oriented cycle or oriented path by $C$. Also, non-tight elements do not index rows of $B_{22}$. Taking the preceding facts into account, we can compute $\operatorname{det} B_{22}$ as follows. First multiply by -1 all columns of $B_{22}$ corresponding to arcs that are traversed in the opposite direction by $C$. Next pick any arc in $A$ and replace the corresponding column by the sum of all columns corresponding to arcs contained in $A$. The column resulting from this operation has the form $(0, \ldots, 0, \beta)^{T}$, where $\beta$ denotes the balance of $C^{\circ}$ (this holds whether $C$ is an oriented cycle or an oriented path). Because the matrix obtained from $B_{22}$ by removing the last row is totally unimodular, we have $\operatorname{det} B=\operatorname{det} B_{22}= \pm \beta$.

If $S^{\circ}$ contained two distinct oriented cycles then it would have two arcs, say $\left(i_{1}, j_{1}\right)$ and $\left(i_{2}, j_{2}\right)$, such that $\left(i_{1}, j_{1}\right)$ is an arc of the first oriented cycle and not of the second, and $\left(i_{2}, j_{2}\right)$ is an arc of the second oriented cycle and not of the first. It follows that when computing $\operatorname{det} B_{22}$ we may replace the column corresponding to $f^{-1}\left(\left(i_{1}, j_{1}\right)\right)$ by a column of the form $\left(0, \ldots, 0, \beta_{1}\right)^{T}$, and then the column corresponding to $f^{-1}\left(\left(i_{2}, j_{2}\right)\right)$ by a column of the form $\left(0, \ldots, 0, \beta_{2}\right)^{T}$. This implies $\operatorname{det} B=\operatorname{det} B_{22}=0$, a contradiction. The result follows.

Lemma 6.2 implies that a vertex of the flow polytope is integral whenever its reduced support is weakly acyclic or contains an oriented cycle of balance \pm 1 . Hence, by Corollary 5.3 (a corollary to the oriented cycle balance lemma), all vertices of the flow polytope are integral provided the semiorder $P$ is reduced. However, the Example shows that the flow polytope can have non-integral vertices. On the positive side, Theorem 6.4 below asserts that $(\mathcal{D})$ always has an integral optimal solution.

REMARK 6.1. It would be natural to call $(\mathcal{P})$ a parametric potential problem. Although parametric versions of some combinatorial optimization problems have been thoroughly studied (see, e.g., Young, Tarjan and Orlin [27] or Agarwal, Eppstein, Guibas and Henzinger [1]), it does not seem to be the case for potential problems. Some particular parametric potential problems have been studied before, for instance in connection with problems whose constraint matrix has the circular ones property (see, e.g., Bartholdi, Orlin and Ratliff [6], Eisenbrand, Oriolo, Stauffer and Ventura [11] or Gijswijt [15]). To the best of our knowledge, we lack a characterization of the parametric potential problems with a totally dual integral system of constraints.

ExAmple 6.1. Consider the semiorder $P$ described in Figure 6.1 by one of its step tableaus, and also by its fringe graph (the denomination of the elements refers to the proof of Theorem 6.4). In the primal linear program $\mathcal{P}$ related to $P$, we set 
$w_{i^{+}}=+1$ and $w_{i^{-}}=-1$ for $i=1,2,3$ and moreover $\omega=2$.
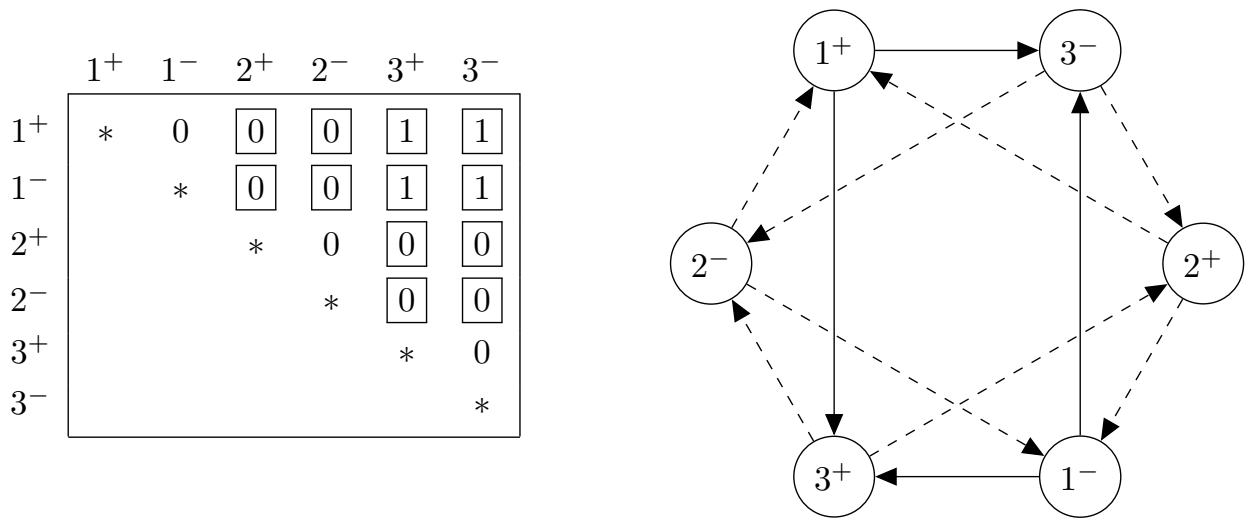

Fig. 6.1. The semiorder $P$ used in Example 6.1 is given here by a step tableau on the left and the fringe graph on the right.

The resulting flow polytope $\mathcal{F}$ has non-integral vertices. Even worse, some of the latter are optimal solutions of $(\mathcal{D})$ : Figure 6.2 provides such an optimal, non-integral vertex $y$ on the left while on the right it gives a first modification $y^{\prime}$ of the latter vertex towards an integral optimal solution of $(\mathcal{D})$ (notations are as in the proof of Theorem 6.4).
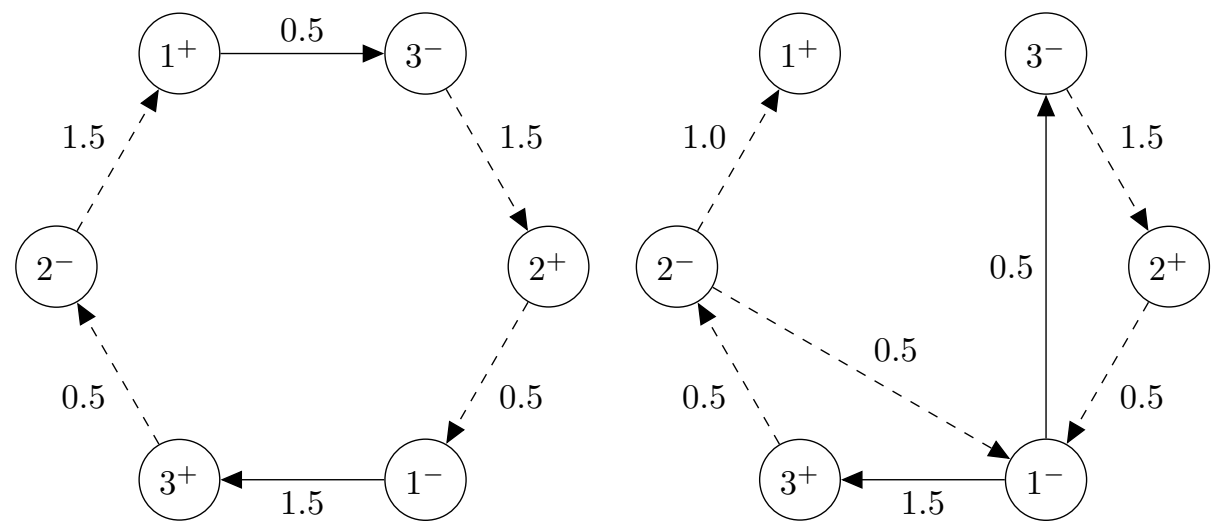

FIG. 6.2. An extremal optimal dual solution y (to the left) and the corresponding optimal dual solution $y^{\prime}$ (to the right) for the dual linear program $\mathcal{D}$ related to the semiorder of Figure 6.1 (see also Example 6.1 and the proof of Theorem 6.4). Arcs with weight zero are not displayed here.

Lemma 6.3. Assume $P$ has two equivalent elements $j$ and $k$ such that $w_{j} \cdot w_{k} \geq 0$. Let $P^{\prime}$ denote the semiorder on $X^{\prime}:=X \backslash\{j, k\} \cup\{\ell\}$ obtained from $P$ by merging $j$ and $k$ to a new element named $\ell$. We denote the nose set and hollow set of $P^{\prime}$ by $N^{\prime}$ and $H^{\prime}$ respectively. Now let $w_{i}^{\prime}:=w_{i}$ for $i \neq \ell, w_{\ell}^{\prime}:=w_{j}+w_{k}$, and $\omega^{\prime}:=\omega$. Let $\left(\mathcal{P}^{\prime}\right)$ and $\left(\mathcal{D}^{\prime}\right)$ denote the linear programs obtained from $(\mathcal{P})$ and $(\mathcal{D})$ by substituting $X^{\prime}, N^{\prime}, H^{\prime}, w^{\prime}$ and $\omega^{\prime}$ for $X, N, H, w$ and $\omega$, respectively. If $\left(\mathcal{D}^{\prime}\right)$ has an optimal integral solution then $(\mathcal{D})$ has an optimal integral solution.

Proof. Assume that $\left(\mathcal{D}^{\prime}\right)$ has an optimal integral solution $y^{\prime}$. Because of $w_{j} \cdot w_{k} \geq$ 0 , we may add the constraint $x_{j}=x_{k}$ to the linear program $(\mathcal{P})$ without changing 
its optimum value. It follows that the optimum values of $(\mathcal{P})$ and $\left(\mathcal{P}^{\prime}\right)$ coincide. By linear programming duality, the optimum values of $(\mathcal{D})$ and $\left(\mathcal{D}^{\prime}\right)$ coincide. Therefore, it suffices to show that $y^{\prime}$ can be transformed into an integral solution $y$ of $(\mathcal{D})$ with the same objective value.

Now consider the quantity $\Delta:=y^{\prime}\left(\delta^{-}(\ell)\right)-y^{\prime}\left(\delta^{+}(\ell)\right)$; we have $\Delta \leq w_{j}+w_{k}$ (the flow balance inequality at $\ell$ ).

First assume $\Delta \geq 0$. Because $w_{j}$ and $w_{k}$ are integral (recall that we assume $w \in \mathbb{Z}^{X}$ and $\omega \in \mathbb{Z}$ for the whole section) and in the present case both nonnegative, we can write $\Delta$ as the sum of two nonnegative integers $\phi_{j}^{-}$and $\phi_{k}^{-}$such that $\phi_{j}^{-} \leq w_{j}$ and $\phi_{k}^{-} \leq w_{k}$. By changing the name of vertex $\ell$ to $j$ we obtain a flow $y$ in the fringe graph of $P$. We ensure $y$ is feasible for $(\mathcal{D})$ by repeating $\phi_{k}^{-}$times the following operation: Find an arc $(i, j)$ with head $j$ such that $y_{i j}>0$, decrease $y_{i j}$ by one unit and increase $y_{i k}$ by one unit.

Second assume $\Delta<0$. In this case, $w_{j}$ and $w_{k}$ are either both nonpositive or both nonnegative and we can write $-\Delta$ as the sum of two nonnegative integers $\phi_{j}^{+}$ and $\phi_{k}^{+}$such that $-\phi_{j}^{+} \leq w_{j}$ and $-\phi_{k}^{+} \leq w_{k}$. To define $y$ we proceed similarly as in the case $\Delta \geq 0$, this time modifying the flow values on arcs with tail $j$.

In both cases, the flow $y$ remains integral and keeps the same objective value during the whole modification process. Moreover, the final flow $y$ is feasible for $(\mathcal{D})$. It follows that $y$ is an integral optimal solution of $(\mathcal{D})$. This concludes the proof. $\square$

Theorem 6.4 (Total dual integrality). For each $w \in \mathbb{Z}^{X}$ and $\omega \in \mathbb{Z}$ such that $(\mathcal{P})$ is bounded, $(\mathcal{D})$ has an integral optimum solution. In particular, when $\varepsilon$ is rational, the system (3.1) defining the representation polyhedron $\mathcal{R}_{\varepsilon}$ of $P$ is totally dual integral.

Proof. By Lemma 6.3, we may assume the following:

Each equivalence class of $P$ contains either one element, or two elements $j$ and $k$ such that $w_{j} \cdot w_{k}<0$.

Indeed, suppose that $(\star)$ does not hold. After repeatedly merging pairs of equivalent elements $j, k$ such that $w_{j} \cdot w_{k} \geq 0$, we obtain a semiorder and a cost vector satisfying $(\star)$. If the corresponding dual linear program has an integral optimal solution, so does the original dual linear program $(\mathcal{D})$.

From now on, we assume that $(\star)$ holds. We use this to define a linear ordering $\leq$ contained in the trace of $P$. The linear ordering $\leq$ is the unique linear ordering compatible with $P$ such that $i^{+}<i^{-}$whenever $i^{+}$and $i^{-}$are two equivalent elements with $w_{i^{+}}>0$ and $w_{i^{-}}<0$.

Consider any extremal optimal solution $v$ of $(\mathcal{D})$. Let $S^{\circ}$ denote the reduced support of $v$. By Lemma 6.2, $S^{\circ}$ contains at most one oriented cycle. Let $\gamma$ denote the length of the oriented cycle in $S^{\circ}$ if one exists; otherwise let $\gamma=0$. Assume that the optimal integral solution $v$ is chosen in such a way that $\gamma$ is minimum. We claim that $v$ is integral.

By Lemma 6.2, the claim holds when $\gamma=0$. Assume now that $\gamma>0$ and let $C^{\circ}$ denote the unique oriented cycle contained in $S^{\circ}$. If $\gamma=1$ then $C^{\circ}$ is a loop and has balance \pm 1 . If $\gamma=2$ then $C^{\circ}$ has exactly two arcs, say $a_{1}$ and $a_{2}$, that are either parallel or anti-parallel. If $a_{1}$ and $a_{2}$ are parallel, by Lemma 6.1, we have either $f^{-1}\left(a_{1}\right), f^{-1}\left(a_{2}\right) \in N$ or $f^{-1}\left(a_{1}\right), f^{-1}\left(a_{2}\right) \in H^{-1}$. Thus $C^{\circ}$ has balance 0 . If $a_{1}$ and $a_{2}$ are anti-parallel, the minimality of $\gamma$ is contradicted. Indeed, consider the optimal dual solution $y^{\prime}$ obtained from $v$ by decreasing the flow simultaneously on $f^{-1}\left(a_{1}\right)$ and $f^{-1}\left(a_{2}\right)$ until one of the two corresponding flow values is zero. Let now $v^{\prime}$ be any extremal optimal dual solution satisfying all flow balance or trivial equalities 
$y^{\prime}$ satisfies. Then the reduced support of $v^{\prime}$ is contained in that of $y^{\prime}$. Hence the reduced support of $v^{\prime}$ has no oriented cycle, which is the desired contradiction to the minimality of $\gamma$. Therefore, whenever $\gamma \in\{1,2\}$, the balance of $C^{\circ}$ is in $\{-1,0,+1\}$. By Lemma 6.2, we conclude that $v$ is integral, and thus that the claim holds, for those values of $\gamma$. Henceforth, we assume $\gamma \geq 3$. In particular this implies that $S^{\circ}$ is simple (that is, has no loop, and no pair of parallel or antiparallel arcs).

We say that an unordered pair $\left\{j^{+}, j^{-}\right\}$of equivalent elements with $w_{j^{+}}>0$ and $w_{j^{-}}<0$ is bad if the following conditions hold:

(i) $j^{+}$and $j^{-}$are both vertices of $C^{\circ}$,

(ii) one of the arcs of $C^{\circ}$ has tail $j^{+}$, or head $j^{-}$.

Condition (i) implies in particular that one of $j^{+}$and $j^{-}$is tight with respect to $v$. Otherwise, $j^{+}$and $j^{-}$are contracted to the special vertex $\tau$ when $S$ is transformed in $S^{\circ}$, and are thus not vertices of $C^{\circ}$.

First assume that $v$ has no bad pair. Let $Z$ denote the set of elements that are not tight with respect to $v$. By Lemma $6.1, Z$ is contained in the initial class of $P$. Moreover, $(\star)$ implies that $Z$ has either one element or two elements constituting the initial class of $P$. We obtain a new semiorder $P^{\prime}$ from $P$ as follows. If $Z$ contains more than one vertex, merge the two elements in $Z$ to a new element named $\tau$, exactly as the special vertex of $S^{\circ}$. Next (in either case for $|Z|$ ) delete all elements not contained in $C^{\circ}$. This concludes the construction of $P^{\prime}$.

Let $\leq{ }^{\prime}$ denote the linear ordering on the ground set of $P^{\prime}$ deduced from $\leq$. Let $M^{\prime}$ be the tableau of $P^{\prime}$ indexed by the linear ordering $\leq^{\prime}$. The key observation is that $C^{\circ}$ can be regarded as an oriented cycle in the fringe graph of the tableau $M^{\prime}$. This follows from our careful choice of compatible linear ordering $\leq$ and the assumption that $v$ has no bad pair, see the next paragraph for details. By the oriented cycle balance lemma (Lemma 5.2), the balance of $C^{\circ}$ is in $\{-1,0,+1\}$. Lemma 6.2 then implies that $v$ is integral and hence the claim holds in case $v$ has no bad pair.

We verify that our key observation holds. By construction, the vertex set of $C^{\circ}$ and the ground set of $P^{\prime}$ coincide. Consider some $\operatorname{arc}(a, b)$ contained in $C^{\circ}$. Assume first that $f^{-1}((a, b))$ is a nose of $P$. By Lemma $4.1,(a, b)$ is a nose of $P^{\prime}$. Let $C_{a}^{\prime}$ and $C_{b}^{\prime}$ denote the equivalence classes of $a$ and $b$ with respect to $P^{\prime}$. Because $v$ has no bad pair, $a$ is maximal in $C_{a}^{\prime}$ and $b$ is minimal in $C_{b}^{\prime}$, with respect to $\leq^{\prime}$. Indeed, if $a$ is not maximal in $C_{a}^{\prime}$ then $C_{a}^{\prime}=\left\{a^{+}, a^{-}\right\}$for some pair of elements with $w_{a^{+}}>0$, $w_{a^{-}}<0$ and $a=a^{+}$. Then $\left\{a^{+}, a^{-}\right\}$is a bad pair because $a^{+}$and $a^{-}$are both vertices of $C^{\circ}$ and $(a, b)=\left(a^{+}, b\right)$ is an arc of $C^{\circ}$ with tail $a^{+}$. This contradicts our assumption on $C^{\circ}$. Similarly, if $b$ is not minimal in $C_{b}^{\prime}$ then $C_{b}^{\prime}=\left\{b^{+}, b^{-}\right\}$for some pair of elements with $w_{b^{+}}>0, w_{b^{-}}<0$ and $b=b^{-}$. Then $\left\{b^{+}, b^{-}\right\}$is a bad pair because $b^{+}$and $b^{-}$are both vertices of $C^{\circ}$, and $(a, b)=\left(a, b^{-}\right)$is an $\operatorname{arc}$ of $C^{\circ}$ with head $b^{-}$, a contradicition. It follows that $(a, b)$ is a nose of $M^{\prime}$. A similar argument shows that if $(c, d)$ is contained in $C^{\circ}$ and $f^{-1}((d, c))$ is a hollow inverse of $P$, then $(d, c)$ is hollow inverse of $M^{\prime}$.

Now suppose that $v$ has a bad pair $\left\{j^{+}, j^{-}\right\}$. We show that in this situation there exists another extremal optimal solution $v^{\prime}$ of $(\mathcal{D})$ whose reduced support has no oriented cycle of length $\gamma$ or more, contradicting the minimality of $\gamma$.

Without loss of generality we may assume that some $\operatorname{arc}$ of $C^{\circ}$ has tail $j^{+}$because the other case, occurring when some arc of $C^{\circ}$ has head $j^{-}$, is similar. Thus there is an element $k$ such that $\left(j^{+}, k\right)$ is an arc of the oriented cycle $C$. In the next two paragraphs, we explain how to reroute the flow going through $\left(j^{+}, k\right)$ in such a way as to obtain a new optimal dual solution $y^{\prime}$. Take any extremal optimal dual solution $v^{\prime}$ 
satisfying all flow balance or trivial equalities this solution $y^{\prime}$ satisfies; then $v^{\prime}$ provides the extremal optimal solution $v^{\prime}$ we are looking for. To construct the solution $y^{\prime}$, we consider two cases.

Case 1: The oriented cycle $C^{\circ}$ has an arc with head $j^{+}$(this case occurs in Example 6.1). Let $\left(i, j^{+}\right)$denote this arc. Because $\gamma \geq 3$, the vertices $i$ and $k$ are distinct. We construct the new optimal dual solution $y^{\prime}$ by first initializing $y^{\prime}$ to $v$. Let $\mu:=\min \left\{y_{i j^{+}}^{\prime}, y_{j^{+}}^{\prime}\right\}$. If $j^{-}$is distinct from $i$ and $k$, we modify $y^{\prime}$ by decreasing the flow on $\left(i, j^{+}\right)$and $\left(j^{+}, k\right)$ by $\mu$ and then increasing the flow on $\left(i, j^{-}\right)$and $\left(j^{-}, k\right)$ by $\mu$. Else $j^{-}=i$ or $j^{-}=k$, we decrease the flow on $\left(i, j^{+}\right)$and $\left(j^{+}, k\right)$ by $\mu$ and then increase the flow on $(i, k)$ by $\mu$.

Case 2: The oriented cycle $C^{\circ}$ has no arc with head $j^{+}$. Then $C^{\circ}$ has a second arc with tail $j^{+}$, say $\left(j^{+}, \ell\right)$. Because $\gamma \geq 3$, the vertices $\ell$ and $k$ are distinct. If $j^{+}$is not tight with respect to $v$ then $j^{-}$and all the other elements of $X$ are tight and we can make $j^{+}$tight by redefining $w_{j+}$ as follows:

$$
w_{j^{+}}:=-\sum_{\substack{i \in X \\ i \neq j^{+}}} w_{i} .
$$

This preserves the fact that $v$ is an extremal optimal dual solution. If $j^{+}$is tight, because $w_{j^{+}} \geq 0$, there is some flow coming into $j^{+}$. We construct the new optimal dual solution $y^{\prime}$ by first initializing $y^{\prime}$ to $v$. If $j^{-}$is distinct from $k$ and $\ell$, we then repeat the following steps as long as one of $\left(j^{+}, k\right)$ and $\left(j^{+}, \ell\right)$ is in the support of $y^{\prime}$ : pick some arc $\left(i, j^{+}\right)$in the support of $y^{\prime}$ with head $j^{+}$, compute $\mu:=\min \left\{y_{i j^{+}}^{\prime}, \max \left\{y_{j^{+}}^{\prime}, y_{j^{+} \ell}^{\prime}\right\}\right.$, decrease $y_{i j^{+}}^{\prime}$ and $y_{j^{+} k}^{\prime}$ (resp. $y_{j^{+} \ell}^{\prime}$ if $y_{j^{+} k}^{\prime}<\mu$ ) by $\mu$, and increase $y_{i j^{-}}^{\prime}$ and $y_{j^{-k}}^{\prime}$ (resp. $\left.y_{j-\ell}^{\prime}\right)$ by $\mu$. Else if $j^{-}=k$ or $j^{-}=\ell$, we repeatedly pick some $\operatorname{arc}\left(i, j^{+}\right)$in the support of $y^{\prime}$ with head $j^{+}$, compute $\mu:=\min \left\{y_{i j^{+}}^{\prime}, y_{j^{+} j^{-}}^{\prime}\right\}$, decrease $y_{i j^{+}}^{\prime}$ and $y_{j^{+} j^{-}}^{\prime}$ by $\mu$, and increase $y_{i j^{-}}^{\prime}$ by $\mu$, until $\left(j^{+}, j^{-}\right)$leaves the support of $y^{\prime}$.

We leave it to the reader to check that in the two cases considered above every oriented cycle in the reduced support of $y^{\prime}$ has length strictly smaller than $\gamma$.

Finally, let $v^{\prime}$ be any extremal optimal dual solution satisfying all flow balance or trivial equalities $y^{\prime}$ satisfies. By construction, either $v^{\prime}$ contains no oriented cycle in its reduced support or the length of the unique oriented cycle in the reduced support of $v^{\prime}$ is strictly smaller than $\gamma$. This contradicts our choice of $v$. Our second claim, and hence the theorem, follows.

Because total dual integrality has many consequences (see, e.g., Schrijver [24]), Theorem 6.4 implies several properties of the representation polyhedron $\mathcal{R}_{\varepsilon}$. First, when $\varepsilon$ is an integer, all the vertices of $\mathcal{R}_{\varepsilon}$ are integral. By Proposition 1.1, it follows that for any positive real $\varepsilon$, all vertex coordinates of $\mathcal{R}_{\varepsilon}$ are integral multiples of $\varepsilon$. Second, we derive from Theorem 6.4 a stronger result. A polyhedron $Q$ in $\mathbb{R}^{d}$ is integral if $Q=\operatorname{conv}\left(Q \cap \mathbb{Z}^{d}\right)$.

COROLlaRY 6.5. For all positive integers $\varepsilon$, the representation polyhedron $\mathcal{R}_{\varepsilon}$ is integral.

The next two results use the following fact. First, every $\varepsilon$-representation $(x, r)$ of $P$ can be projected to an $\varepsilon$-representation $(\bar{x}, r)$ of the reduced quotient $P / \sim$ (cf. Section 2): We let $\bar{x}_{I}:=\min \left\{x_{j}: j \in I\right\}$ for all equivalence classes $I \in X / \sim$. The projected representation $(\bar{x}, r)$ has the same threshold as $(x, r)$ and satisfies $\bar{x}_{I} \leq x_{j}$ for all $j \in I \in X / \sim$. Second, every $\varepsilon$-representation $(\bar{x}, r)$ of $P / \sim$ can be canonically lifted to an $\varepsilon$-representation $(x, r)$ of $P$ with the same threshold by letting $x_{j}:=\bar{x}_{I}$ for all $j \in I \in X / \sim$. 
Corollary 6.6 (Minimum threshold). Let $\rho^{*}$ be the maximum number of noses in a directed cycle of the fringe graph of $P / \sim$ and let $r^{*}=\varepsilon \rho^{*}$. Then for all $\varepsilon-$ representations $(x, r)$ of $P$ we have $r^{*} \leq r$.

Proof. Without loss of generality, $P$ may be assumed to be reduced. Thus we have $P=P / \sim$.

Let $w=0$ and $\omega=1$. Then $(\mathcal{P})$ is bounded and by Theorem 6.4 the dual $(\mathcal{D})$ has an integral optimal solution $y$. Because $y$ is feasible and $w$ is zero, $y$ is necessarily a circulation (for background about circulations, we refer the reader to, e.g., [16]). Because $y$ is integral, it admits a decomposition as a sum of characteristic vectors of directed cycles in $D(P)$.

It follows from the feasibility of $y$ and Corollary 5.4 that the decomposition of $y$ involves at most one cycle, since otherwise $y$ violates the threshold constraint. By the optimality of $y$, this cycle (if it exists) contains exactly $\rho^{*}$ noses. In all cases, we have $\varepsilon y(N)=\varepsilon \rho^{*}=r^{*}$. Now by linear programming duality we conclude that $r^{*}$ is the minimum threshold across all $\varepsilon$-representations. $\square$

We now provide a different proof for the central result of Pirlot [20].

Corollary 6.7 (Minimal $\varepsilon$-representation). There exists a (unique) $\varepsilon$-representation $\left(x^{*}, r^{*}\right)$ of $P$ such that for all $\varepsilon$-representations $(x, r)$ of $P$ with the same $\varepsilon$ we have $r^{*} \leq r$ and also $x_{i}^{*} \leq x_{i}$ for all $i \in X$.

Proof. As in the previous proof, it is not restrictive to assume that the semiorder $P$ is reduced.

Let again $\rho^{*}$ denote the maximum number of noses in a directed cycle of the fringe graph. We define the length of an $\operatorname{arc}(i, j)$ of the fringe graph to be $\rho^{*}$ if $(i, j)$ is a hollow inverse or $-\rho^{*}-1$ if $(i, j)$ is a nose. By Corollary 5.4, the fringe graph has no negative length cycles with respect to these arc lengths.

Now let $m$ denote the first element in the trace. For all $i \in X$, define $x_{i}^{*}$ to be $-\varepsilon$ times the distance from $m$ to $i$ in the fringe graph. In other words, let

$$
\begin{aligned}
x_{i}^{*} & =-\varepsilon \min \left\{\rho^{*} \chi^{R}\left(H^{-1}\right)-\left(\rho^{*}+1\right) \chi^{R}(N): R \text { is an } m-i \text { directed path }\right\} \\
& =\varepsilon \max \left\{\left(\rho^{*}+1\right) \chi^{R}(N)-\rho^{*} \chi^{R}\left(H^{-1}\right): R \text { is an } m-i \text { directed path }\right\},
\end{aligned}
$$

where $\chi^{R}$ denotes the characteristic vector of $R$. Moreover, let $r^{*}=\varepsilon \rho^{*}$.

We claim that $\left(x^{*}, r^{*}\right)$ is an $\varepsilon$-representation. Consider first a nose $(a, b)$ of $P$. From the definition of $x^{*}$, we get $x_{b}^{*} \geq x_{a}^{*}+\varepsilon\left(\rho^{*}+1\right)=x_{a}^{*}+r^{*}+\varepsilon$. Next let $(a, b)$ be a hollow inverse of $P$. Then we have $x_{b}^{*} \geq x_{a}^{*}-\varepsilon \rho^{*}=x^{*}+b-r^{*}$. Finally, note that it follows from Lemma 4.5 that $x^{*}$ is nonnegative. So the claim holds.

Finally, consider any $\varepsilon$-representation $(x, r)$. By Corollary 6.6 we have $r^{*} \leq r$. Now fix $i$ and let $R$ be an $m-i$ directed path of minimum length. By summing all inequalities in (3.1) corresponding to arcs of $R$ and the inequality $x_{m} \geq 0$, we get

$$
\begin{aligned}
x_{i} & \geq(r+\varepsilon) \chi^{R}(N)-r \chi^{R}\left(H^{-1}\right)=\varepsilon \chi^{R}(N)+r\left(\chi^{R}(N)-\chi^{R}\left(H^{-1}\right)\right) \\
& \geq \varepsilon \chi^{R}(N)+r^{*}\left(\chi^{R}(N)-\chi^{R}\left(H^{-1}\right)\right)=x_{i}^{*} .
\end{aligned}
$$

The second inequality follows from the fact that, by Lemma 5.5, the directed path $R$ has more noses than hollow inverses and the inequality $r \geq r^{*}$ (Corollary 6.6).

Notice that the minimal representation $\left(x^{*}, r^{*}\right)$ as in Corollary 6.7 always provides a vertex of the polyhedron $\mathcal{R}_{\varepsilon}$. There are semiorders for which it is the only vertex. However, by using porta [7], we found examples of semiorders having (i) several vertices all with threshold equal to $r^{*}$, (ii) having one vertex with threshold $r^{*}$ and one other vertex with another threshold, (iii) having one vertex with threshold $r^{*}$ and several other vertices (with equal or varying thresholds), (iv) having several vertices with threshold $r^{*}$ and several additional vertices (with equal or varying thresholds). 
7. Extreme rays. In this section we characterize the extreme rays of the representation polyhedron $\mathcal{R}_{\varepsilon}$ of a semiorder $P$ on $X$. As usual, we assume that $P$ has trace $T$, nose set $N$ and hollow set $H$.

Recall that the extreme rays of a polyhedron coincide with the extreme rays of its characteristic cone. Moreover, if $A x \geq b$ is some linear description of the polyhedron then $A x \geq 0$ is a linear description of its characteristic cone (see, e.g., [23, 28]). Letting $\mathcal{C}$ denote the characteristic cone of $\mathcal{R}_{\varepsilon}$, we infer from Proposition 3.3 that $\mathcal{C}$ is described by the following linear constraints:

$$
\begin{array}{rll}
-x_{a}+x_{b}-r & \geq 0, & \forall(a, b) \in N, \\
-x_{d}+x_{c}+r & \geq 0, & \forall(d, c) \in H^{-1}, \\
x_{i} \geq 0, & \forall i \in X, \\
r & \geq 0 .
\end{array}
$$

Note that the system does not depend on $\varepsilon$ (being homothetic, all polyhedra $\mathcal{R}_{\varepsilon}$ have the same characteristic cone $\mathcal{C}$ ).

It turns out that the extreme rays of $\mathcal{R}_{\varepsilon}$ are tightly related to the strongly connected components of the fringe graph of $P$. By results of Section 4, these are essentially maximal intervals in $T$ inducing no short nose. More precisely, as stated in Lemma 4.3, a subset of $X$ is a strongly connected component of $D(P)$ if and only if it is the union of all classes of $P$ between some leading class and the next trailing class. Below, we call a strongly connected component initial if it contains the initial class.

Proposition 7.1. Let $P$ be a semiorder on $X$ with trace $T$. The extreme rays of $\mathcal{R}_{\varepsilon}$ are precisely the rays generated by the vectors $(z, q)$ in $\mathbb{R}^{X} \times \mathbb{R}$ that are one of the following two types:

(A) $q=0, z \in\{0,1\}^{X}, z_{i} \leq z_{j}$ whenever $i T j$, and $z$ constant on each strongly connected component of the fringe graph $D(P)$ without being the zero vector;

(B) $q=1$ and $z \in \mathbb{Z}_{+}^{X}$ satisfies

(a) $z_{m}=0$ for some initial element $m$,

(b) $z_{a}+1 \leq z_{b}$ for all $(a, b) \in N$,

(c) $z_{d}-1 \leq z_{c}$ for all $(d, c) \in H^{-1}$,

(d) for each strongly connected component of $D(P)$ that is not initial there is a short nose $(a, b)$ with its head in the component such that $z_{a}+1=z_{b}$.

Proof. For $t \in\{0,1\}$, let $\mathcal{Q}_{t}:=\{(x, r) \in \mathcal{C}: r=t\}$ where $\mathcal{C}$ is the characteristic cone of $\mathcal{R}_{\varepsilon}$. From general theory on polyhedra we know that each extreme ray of $\mathcal{Q}_{0}$ is an extreme ray of $\mathcal{C}$, and that each vertex of $\mathcal{Q}_{1}$ generates an extreme ray of $\mathcal{C}$. Conversely, all extreme rays of $\mathcal{C}$ can be obtained in such a way. Finally, as recalled above, the extreme rays of $\mathcal{R}_{\varepsilon}$ coincide with those of $\mathcal{C}$.

First we show that the extreme rays of $\mathcal{Q}_{0}$ are precisely the rays generated by the vectors $(z, q)$ satisfying $(\mathrm{A})$. Let $(i, j)$ denote any arc of $D(P)$. By replacing $r$ with 0 in the inequality from (7.1) corresponding to $(i, j)$ we obtain $x_{i} \leq x_{j}$. Hence $\mathcal{Q}_{0}$ is the set of all points $(x, r)$ with $r=0$, and $x$ nonnegative, nondecreasing with respect to $T$ and constant on each strongly connected component of $D(P)$. The extreme rays of $\mathcal{Q}_{0}$ are then as in (A). This concludes the first part of the proof.

Second we show that the vertices of $\mathcal{Q}_{1}$ are precisely the points $(z, q)$ satisfying (B). We start by proving that every vertex of $\mathcal{Q}_{1}$ satisfies (B). By replacing $r$ with 1 in all equations of (7.1) except the equation $r \geq 0$, replacing the latter equation by $r=1$ and moving all constants to the right hand side we obtain a system defining $\mathcal{Q}_{1}$. Since this system has a totally unimodular constraint matrix and integral right hand sides, we conclude that all vertices of $\mathcal{Q}_{1}$ are integral. 
Consider a vertex $v=(z, q)$ of $\mathcal{Q}_{1}$. We have $q=1$ and, by what precedes, $z_{i} \in \mathbb{Z}_{+}$ for all $i \in X$; also, (b) and (c) are satisfied. If $v$ fails to fulfil (a) or (d), then there exists a vector $g=(z, q)$ as in (A) such that $v \pm \sigma g$ belongs to $\mathcal{Q}_{1}$ for some positive real $\sigma$, contradicting the fact that $v$ is a vertex. It follows that every vertex of $\mathcal{Q}_{1}$ satisfies (B).

Next we prove that every point $v=(z, q)$ satisfying (B) is a vertex of $\mathcal{Q}_{1}$. We proceed by showing the following for any point $v^{\prime}=\left(z^{\prime}, q^{\prime}\right)$ of $\mathcal{Q}_{1}$ : Whenever $v^{\prime}$ satisfies with equality all valid inequalities for $\mathcal{Q}_{1}$ that $v$ satisfies with equality, then $v^{\prime}$ equals $v$.

We now gather some useful valid inequalities for $\mathcal{Q}_{1}$. Consider any two elements $i$ and $j$. By Lemma 4.5, the inequality $-x_{i}+x_{j} \geq 0$ is valid whenever $i T j$ and $\neg j T i$. Next assume $i \sim j$. If $P$ has only one equivalence class then $(i, j) \in H^{-1}$ and the inequality $-x_{i}+x_{j} \geq-1$ is valid. Otherwise, by Lemma 4.3 , there exists a directed cycle in the fringe graph of $P / \sim$ passing through $C_{i}=C_{j}$. By Corollary 5.4, this cycle has exactly one more hollow inverse than noses. By interpreting the directed cycle as a directed path from $i$ to $j$ in the fringe graph of $P$, we again conclude that $-x_{i}+x_{j} \geq-1$ is valid.

Define a relation $T^{\star}$ by letting (for any elements $i, j$ in $X$ ) $i T^{\star} j$ if and only if $i T j$ and $z_{i} \leq z_{j}$. Because $i T j$ and $\neg j T i$ imply $-z_{i}+z_{j} \geq 0$, that is, $z_{i} \leq z_{j}$, the relation $T^{\star}$ is a weak order refining $T$. Because $i \sim j$ implies $\left|z_{i}-z_{j}\right| \leq 1$ and $z$ is integral, each equivalence class of $T$ is split into at most two equivalence classes of $T^{\star}$.

Since $v$ and $v^{\prime}$ are both in $\mathcal{Q}_{1}$, we have $q^{\prime}=q=1$. We show by induction on the rank of an element $j$ with respect to $T^{\star}$ that $z_{j}=z_{j}^{\prime}$ for all $j$ in $X$. By (B.a) we have $z_{j}=0$ and thus $z_{j}^{\prime}=0$ if $j$ is initial in $T^{\star}$.

Now assume that $j$ is not initial in $T^{\star}$ and $z_{i}=z_{i}^{\prime}$ holds for all elements $i$ with $i T^{\star} j$ and $\neg j T^{\star} i$. Consider an element $i$ whose equivalence class in $T^{\star}$ comes just before the equivalence class of $j$ in $T^{\star}$. In particular, we have $z_{i} \leq z_{j}$. To conclude the proof, it thus suffices to consider the following four cases. Note that we have $z_{k}=z_{k}^{\prime}$ whenever $k T^{\star} i$ (in particular when $k=i$ ) by the induction hypothesis. This will be used several times below.

CASE 1. $z_{i}=z_{j}$ and $i \sim j$. Then $i$ and $j$ are equivalent in $T^{\star}$, contradicting our choice of $i$. Hence this case cannot occur.

CASE 2. $z_{i}=z_{j}$ and $\neg i \sim j$. Then we have $i T j$ and $\neg j T i$, thus $-x_{i}+x_{j} \geq 0$ is valid for $\mathcal{Q}_{1}$. Because the latter is tight at $v$, it is also tight at $v^{\prime}$. It follows that $z_{j}^{\prime}=z_{i}^{\prime}=z_{i}=z_{j}$.

CASE 3. $z_{i}<z_{j}$ and $i \sim j$. Then we also have $j \sim i$, thus $-x_{j}+x_{i} \geq-1$ is valid. By the integrality of $z$, the latter inequality is tight at $v$ and hence at $v^{\prime}$. Therefore, we get $z_{j}^{\prime}=z_{i}^{\prime}+1=z_{i}+1=z_{j}$.

CASE 4. $z_{i}<z_{j}$ and $\neg i \sim j$. For $U:=\left\{k \in X: k T^{\star} i\right\}$, consider two cases.

If $U$ defines a directed cut in $D(P)$ then $(i, j)$ is a short nose (in view of Lemma 4.3). In this case, (B) implies that the inequality $-x_{i}+x_{j} \geq 1$ (which is valid for $\mathcal{Q}_{1}$ ) is tight at $v$ and hence also at $v^{\prime}$. So we have $z_{j}^{\prime}=$ $z_{i}^{\prime}+1=z_{i}+1=z_{i}$.

If $U$ does not define a directed cut, there is an arc from $X \backslash U$ to $U$, say $(d, c)$. Because $c T^{\star} i$ and $j T^{\star} d$, we have $z_{c} \leq z_{i}<z_{j} \leq z_{d}$. It follows that $(d, c)$ is a hollow inverse. Indeed, otherwise we would have $-z_{d}+z_{c} \geq 1$ and thus $z_{d}<z_{c}$, a contradiction. Because $(d, c)$ is a hollow inverse, we have $z_{d} \leq z_{c}+1$. By integrality of $z$ together with $z_{c} \leq z_{i}<z_{j} \leq z_{d}$, it follows 
that $x_{i}+1 \leq x_{j}$ is tight at $v$. This inequality, which is valid for $\mathcal{Q}_{1}$, is thus also tight at $v^{\prime}$. Therefore we have $z_{j}^{\prime}=z_{i}^{\prime}+1=z_{i}+1=z_{j}$.

Another nice property of $\mathcal{R}_{\varepsilon}$ is related to the integral polyhedron $\mathcal{Q}_{1}$ used in the proof of Proposition 7.1: When the semiorder $P$ is reduced, $Q_{1}$ contains no further integral point than its vertices. This will not be proved here. (Polytopes with the latter property are investigated by Bárány, Howe, and Scarf [3], Bárány and Kantor [4], Bárány, Scarf and Shallcross [5], Deza and Onn [9], etc.)

8. Conclusion. We investigate the polyhedron consisting of all $\varepsilon$-representations of a given semiorder. To this aim, we regard the semiorder as a binary relation, a step tableau, a system of linear inequalities, or a fringe graph (the directed graph of noses and hollow inverses). Our main contribution lies in showing that the minimum linear description for the representation polyhedron is totally dual integral. The proof is based on a property of the oriented cycles in the fringe graph of any strictly upper triangular step tableau. The latter property generalizes a result of Pirlot [20] (in passing, we offer new proofs for several results of Pirlot [20, 21], for instance the existence of a minimal representation).

Several consequences of our main result are drawn, the strongest one being that the representation polyhedron is integral whenever $\varepsilon$ is. Consequently, for any $\varepsilon$, all coordinates of vertices and components of extreme rays are integral multiples of $\varepsilon$. We give a complete polyhedral characterization of the extreme rays.

For the present, we leave open quite natural questions, for instance about the enumeration of vertices and rays. Our data suggest that there should be nice combinatorial characterizations of the vertices and extreme rays of the representation polyhedron. We hope to address these characterizations in future work.

\section{REFERENCES}

[1] P.K. Agarwal, D. Eppstein, L.J. Guibas, and M.R. Henzinger, Parametric and kinetic minimum spanning trees, Proc. 39th Symp. Foundations of Computer Science, IEEE, November 1998, pp. 596-605.

[2] J. Bang-Jensen and G. Gutin, Digraphs, Springer Monographs in Mathematics, Springer-Verlag London Ltd., London, 2001, Theory, algorithms and applications.

[3] I. Bárány, R. Howe, and H.E. Scarf, The complex of maximal lattice free simplices, Math. Programming 66 (1994), no. 3, Ser. A, 273-281.

[4] I. Bárány and J.-M. Kantor, On the number of lattice free polytopes, European J. Combin. 21 (2000), no. 1, 103-110, Combinatorics of polytopes.

[5] I. Bárány, H. E. Scarf, and D. Shallcross, The topological structure of maximal lattice free convex bodies: the general case, Math. Programming 80 (1998), no. 1, Ser. A, 1-15.

[6] J.J. Bartholdi, III, J.B. Orlin, and H.D. Ratliff, Cyclic scheduling via integer programs with circular ones, Oper. Res. 28 (1980), no. 5, 1074-1085.

[7] T. Christof, PORTA - a POlyhedron Representation Transformation Algorithm, version 1.3.2 (1999), written by T. Christof, maintained by A. Loebel and M. Stoer, available at http://www.informatik.uni-heidelberg.de/groups/comopt/software/PORTA/.

[8] V. Chvátal, Linear Programming, W. H. Freeman and Company, New York, 1983.

[9] M. Deza and S. Onn, Lattice-free polytopes and their diameter, Discrete Comput. Geom. 13 (1995), no. 1, 59-75.

[10] J.-P. Doignon and J.-Cl. Falmagne, Well graded families of relations, Discrete Mathematics 173 (1997), 35-44.

[11] F. Eisenbrand, G. Oriolo, G. Stauffer, and P. Ventura, Circular ones matrices and the stable set polytope of quasi-line graphs, Integer programming and combinatorial optimization, Lecture Notes in Comput. Sci., vol. 3509, Springer, Berlin, 2005, pp. 291-305.

[12] P. Fishburn and B. Monjardet, Norbert Wiener on the theory of measurement (1914, 1915, 1921), J. Math. Psych. 36 (1992), no. 2, 165-184. 
[13] P. C. Fishburn, Interval Orders and Interval Graphs, Wiley, New York, 1985.

[14] A. M. H. Gerards, An orientation theorem for graphs, J. Combin. Theory Ser. B 62 (1994), no. 2, 199-212.

[15] D. Gijswijt, Integer decomposition for polyhedra defined by nearly totally unimodular matrices, SIAM J. Discrete Math. 19 (2005), no. 3, 798-806 (electronic).

[16] B. Korte and J. Vygen, Combinatorial optimization, fourth ed., Springer, Berlin, 2008.

[17] R. D. Luce, Semiorders and a theory of utility discrimination, Econometrica 26 (1956), 178191.

[18] B. G. Mirkin, Description of some relations on the set of real-line intervals, Journal of Mathematical Psychology 9 (1972), 243-252.

[19] B. Monjardet, Axiomatiques et propriétés des quasi-ordres, Mathématiques et Sciences Humaines 63 (1978), 51-82.

[20] M. Pirlot, Minimal representation of a semiorder, Theory and Decision 28 (1990), 109-141.

[21] _ Synthetic description of a semiorder, Discrete Applied Mathematics 31 (1991), 299308.

[22] M. Pirlot and P. Vincke, Semiorders: Properties, representations, applications, Kluwer Academic Publishers, Dordrecht, 1997.

[23] A. Schrijver, Theory of linear and integer programming, Wiley-Interscience Series in Discrete Mathematics and Optimization, John Wiley \& Sons, Chichester New York, 1986.

[24] Combinatorial optimization. Polyhedra and efficiency, Algorithms and Combinatorics, 24 (three vol.), Springer-Verlag, Berlin, 2003.

[25] D. Scott and P. Suppes, Foundational aspects of theories of measurement, Journal of Symbolic Logic 23 (1958), 113-128.

[26] H. Sharp, Jr., Enumeration of transitive, step-type relations, Acta Mathematica Academiae Scientiarum Hungaricae $22(1971 / 72), 365-371$.

[27] N.E. Young, R.E. Tarjan, and J.B. Orlin, Faster parametric shortest path and minimum-balance algorithms, Networks 21 (1991), no. 2, 205-221.

[28] G.M. Ziegler, Lectures on polytopes, Graduate Texts in Mathematics, Springer-Verlag, NewYork, 1995. 